\title{
MRI with ultrahigh field strength and high- performance gradients: challenges and opportunities for clinical neuroimaging at 7 $T$ and beyond
}

\author{
Behroze Vachha ${ }^{1}$ and Susie Y. Huang ${ }^{2^{*}}$ (1)
}

\begin{abstract}
Research in ultrahigh magnetic field strength combined with ultrahigh and ultrafast gradient technology has provided enormous gains in sensitivity, resolution, and contrast for neuroimaging. This article provides an overview of the technical advantages and challenges of performing clinical neuroimaging studies at ultrahigh magnetic field strength combined with ultrahigh and ultrafast gradient technology. Emerging clinical applications of 7-T MRI and state-of-the-art gradient systems equipped with up to $300 \mathrm{mT} / \mathrm{m}$ gradient strength are reviewed, and the impact and benefits of such advances to anatomical, structural and functional MRI are discussed in a variety of neurological conditions. Finally, an outlook and future directions for ultrahigh field MRI combined with ultrahigh and ultrafast gradient technology in neuroimaging are examined.
\end{abstract}

Keywords: Epilepsy, Magnetic fields, Magnetic resonance imaging, Multiple sclerosis, Neuroimaging

\section{Key points}

- Increased signal-to-noise ratio and high spatial resolution conferred by ultrahigh field magnetic resonance imaging (MRI) can be used to improve the delineation of small anatomical structures and subtle pathology.

- The challenges associated with ultrahigh field MRI include artifacts associated with increased susceptibility effect, inhomogeneity of the radiofrequency transmit field, and increased radiofrequency energy deposition in tissues.

\footnotetext{
* Correspondence: susie.huang@mgh.harvard.edu

This article belongs to the thematic series entitled "7-Tesla neuro MRI: from research to clinic" (Guest Editors Prof. Luca Roccatagliata (Genoa/Italy) and Mirco Cosottini (Pisa/Italy)).

${ }^{2}$ Athinoula A. Martinos Center for Biomedical Imaging, Department of Radiology, Massachusetts General Hospital, 149 13th Street, Room 2301, Charlestown, MA 02129, USA

Full list of author information is available at the end of the article
}

- High-performance gradient systems equipped with high maximum gradient amplitudes and slew rates enable increased spatial resolution and faster acquisitions.

- Technical limitations of high-performance gradient systems include the biological effects of rapidly switching, large magnetic fields on the human body, which can induce peripheral nerve stimulation.

\section{Background}

The last 20 years have seen a push in the development of magnetic resonance imaging (MRI) scanner technology with remarkable advances in field strength and progress in gradient coil design and performance. Clinical MRI scanners with magnetic field strengths up to $7 \mathrm{~T}$ and gradient strengths up to $80 \mathrm{mT} / \mathrm{m}$ with a peak slew rate of $200 \mathrm{~T} / \mathrm{m} / \mathrm{s}$ have now become the state-of-the-art imaging technology for clinical and research studies of the brain. For neuroimaging, research in ultrahigh magnetic field strength and ultrahigh, ultra-fast gradient 
technology has led to a multitude of benefits for highresolution anatomic, structural, and functional imaging, opening an array of opportunities for changing the way that patients are imaged and evaluated, and providing new insights into the pathophysiology of neurological diseases.

\section{Ultrahigh field MRI}

\section{Technological overview and state-of-the-art}

Since the first ultrahigh field human MRI images were obtained at $8 \mathrm{~T}$ in 1998 [1], ultrahigh field MRI has gained increasing traction worldwide through the development and dissemination of 7-T scanners for human imaging. The promise of ultrahigh field MRI in pushing the limits of sensitivity and spatial resolution, initially demonstrated in the research arena, has been realized through the commercialization of 7-T MRI scanners by the major MRI scanner vendors, culminating in Food and Drug Administration-approved scanners for clinical use in 2017 through the MAGNETOM Terra 7-T MRI scanner (Siemens Healthineers, Erlangen, Germany) and in 2020 through the Signa 7-T MRI scanner (General Electric Healthcare, Chicago, IL, USA). Based on the success of current 7-T and 9.4-T human MRI scanners, concerted efforts are now underway to develop the next generation of ultrahigh field MRI scanners for human use at 10.5- $\mathrm{T}$ and $11.7-\mathrm{T}$, with field strengths up to $20 \mathrm{~T}$ deemed feasible, opening up new opportunities for unprecedented sensitivity and specificity for human imaging [2].

\section{Challenges and opportunities of imaging at higher field}

The benefits and challenges of imaging at higher static magnetic field strength directly follow from the dependence on magnetic field strength of the physical properties of tissues and other magnetic fields involved in image formation. The main advantage of increasing the magnetic field strength for MRI is increased sensitivity. The signal scales greater than linearly with magnetic field strength [3]. The resulting increase in signal-tonoise ratio (SNR) can be used to increase spatial resolution and improve the delineation of small anatomical structures and subtle pathology. For example, the spatial resolution of anatomical T1-weighted images can be pushed to the sub-millimeter level, enabling better estimation of cortical thickness through the reduction of partial volume effects $[4,5]$ and improving the segmentation of the hippocampal subfields $[6,7]$ and amygdalar nuclei $[8,9]$.

Tissue relaxation properties also change with magnetic field strength, with an expected prolongation of the longitudinal relaxation time T1 and shortening of the transverse relaxation times $\mathrm{T} 2$ and $\mathrm{T} 2 *$ at higher field. These changes in relaxation properties along with increased
SNR benefit certain applications; for example, longer T1 relaxation times offer better signal-to-noise ratio for applications such time-of-flight (TOF) magnetic resonance (MR) angiography [10] and arterial spin labeling [11], whereas shorter $\mathrm{T}_{2} *$ relaxation times provide greater image contrast for susceptibility-weighted imaging (SWI) (Fig. 1) and for detecting functional activation by blood oxygenation-level dependent (BOLD) functional MRI (fMRI) [12]. Indeed, a major advantage of imaging at higher field is the improvement in time-series SNR and greater sensitivity to temporal correlations in the BOLD signal, which can capture previously unrecognized nodes in functional networks [13].

The challenges associated with ultrahigh field MRI include increased susceptibility-induced distortions at higher field, inhomogeneity of the radiofrequency (RF) transmit field, and increased RF energy deposition in tissues. As field strength increases, the resonant frequency increases and is approximately $300 \mathrm{MHz}$ at $7 \mathrm{~T}$. Accounting for the dielectric properties of tissue, the wavelength of the RF transmission field at $7 \mathrm{~T}$ is approximately $11 \mathrm{~cm}$, which is shorter than the dimensions of the human body, leading to noticeable variations in the RF transmission field due to the creation of standing waves. Beyond increased transmit inhomogeneity, the amount of RF power deposited in tissue increases with field strength, resulting in higher specific absorption rate (SAR). In practice, clinical 7-T MRI uses only local RF transmit coils, for which the International Electrotechnical Commission has advised a higher threshold of 10 $\mathrm{W} / \mathrm{kg}$ for local SAR compared to the whole-body SAR limit of $2 \mathrm{~W} / \mathrm{kg}$ [14]. The use of MR sequences with high power deposition (e.g., fast spin-echo and inversion-recovery) is limited at higher field strengths. Spatial variations of the RF transmit field due to standing waves can also produce localized heating. Metallic implants may also lead to heating due to RF absorption, although many studies have shown that heating may be less of a problem than initially anticipated, with only minor temperature changes incurred by small, passive implants (e.g., hemostatic clips, aneurysm clips, prosthetic valves, vascular access ports, and ossicular and ocular implants) [15-17].

At higher field strengths, the spectral separation between different chemical species increases, leading to higher spectral resolution, which is beneficial for MR spectroscopy but can lead to greater imaging artifacts due to the increased chemical shift and susceptibility effect. Susceptibility artifacts can adversely affect image quality in ultrahigh field MRI, especially in gradientecho imaging. Susceptibility-induced artifacts occur as a result of microscopic gradients or variations in the magnetic field strength near interfaces of different magnetic susceptibility and result in signal loss and distortion at 


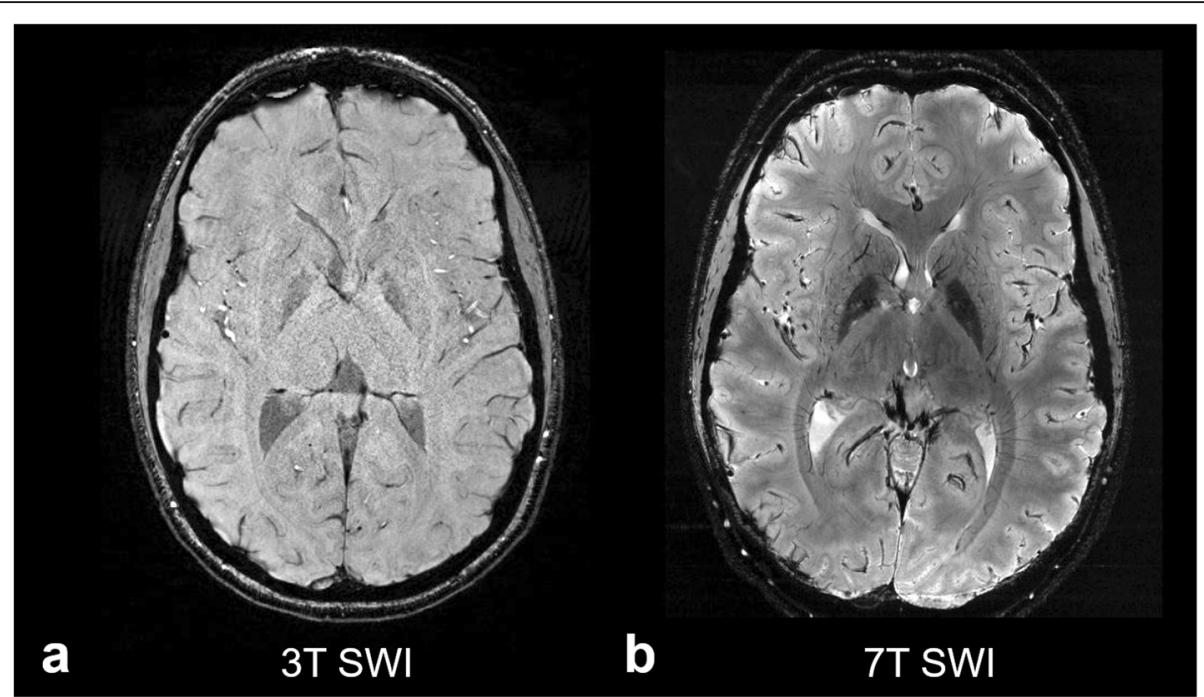

Fig. 1 Comparison of susceptibility-weighted imaging (SWI) using 3-T and 7-T magnetic resonance imaging. Axial SWI images are shown at the level of the basal ganglia in a healthy individual acquired at $3 \mathrm{~T}(\mathbf{a})$ and $7 \mathrm{~T}(\mathbf{b})$. At higher field strength, the shorter T2* relaxation times result in the greater SWI sensitivity to magnetic susceptibility differences in the blood vessels and mineralization in the basal ganglia, particularly in the globus pallidus

air-bone and air-tissue interfaces, which can be particularly pronounced at $7 \mathrm{~T}$ and higher fields. On the other hand, the increased susceptibility effect at higher field may be used for improved diagnostic efficacy, such as increased sensitivity to microhemorrhages and better delineation of the substantia nigra. Finally, while the timeseries SNR increases at higher field, the relative contribution of physiological noise from factors such as cardiac pulsation and respiratory motion also increases at 7 $\mathrm{T}$ compared to lower field strengths [13], necessitating strategies for reducing the effects of physiological noise [18], which are particularly important for fMRI studies at higher field strengths [19].

\section{Clinical applications of ultrahigh field imaging}

The high spatial resolution achievable using ultrahigh field imaging has been used to study various conditions including epilepsy, multiple sclerosis, brain tumors, cerebrovascular diseases, and degenerative disorders. The following sections discuss the current state of research and potential future direction within these clinical fields.

\section{Epilepsy}

In patients with medically refractory epilepsy, surgery may offer the best chances for seizure freedom [20]. Neuroimaging plays a key role in identifying the epileptogenic focus in the presurgical epilepsy work-up. However, approximately one-third of patients with focal epilepsy do not have an identifiable lesion on $1.5-\mathrm{T}$ or 3T MRI [21]. Improved SNR and the exquisitely high spatial resolution conferred by ultrahigh field imaging may help in localizing cryptogenic seizure-onset zones in patients with epilepsy that may have been missed at conventional field strengths [22, 23] (Fig. 2). Feldman et al. demonstrated that 7-T MRI revealed abnormalities of epileptogenic potential in 25 of 37 patients with focal epilepsy who had non-lesional diagnostic $1.5-\mathrm{T}$ or $3-\mathrm{T}$ MRI scans; 15 of these abnormalities corresponded to the clinically suspected seizure-onset site, and the detection of some abnormalities altered subsequent management [24]. SWI sequences at $7 \mathrm{~T}$ in this study were highly sensitive for the detection of developmental venous anomalies, cavernous malformations (Fig. 3), and polymicrogyria (Fig. 4), which either colocalized to electroencephalography data or were associated with suspected site of seizure onset [24]. These findings were supported by a prior study that found a greater number of cavernous malformations using $\mathrm{T} 2$ *-weighted gradient-echo imaging at $7 \mathrm{~T}$ compared to $1.5 \mathrm{~T}$ [25].

In a large cohort of 67 patients with pharmacoresistant epilepsy and nonlesional 3-T MRI undergoing presurgical evaluation, Wang et al. reported that an unaided visual review of 7-T images detected previously unappreciated subtle lesions in $22 \%(15 / 67)$ of patients with the total yield increasing to $43 \%$ when aided by post processing of the $7-\mathrm{T}$ T1-weighted magnetization-prepared two rapid acquisition gradient-echo sequence using the morphometric analysis program (MAP); the majority of lesions detected were focal cortical dysplasia [26]. The authors reported that 7-T MAP yielded 25\% more lesions than 3-T MAP and demonstrated increased conspicuity in 46\% [26].

Asymmetric distribution of perivascular spaces was reported in epilepsy patients compared to healthy controls when quantified on 7-T MRI scans. In the majority of 


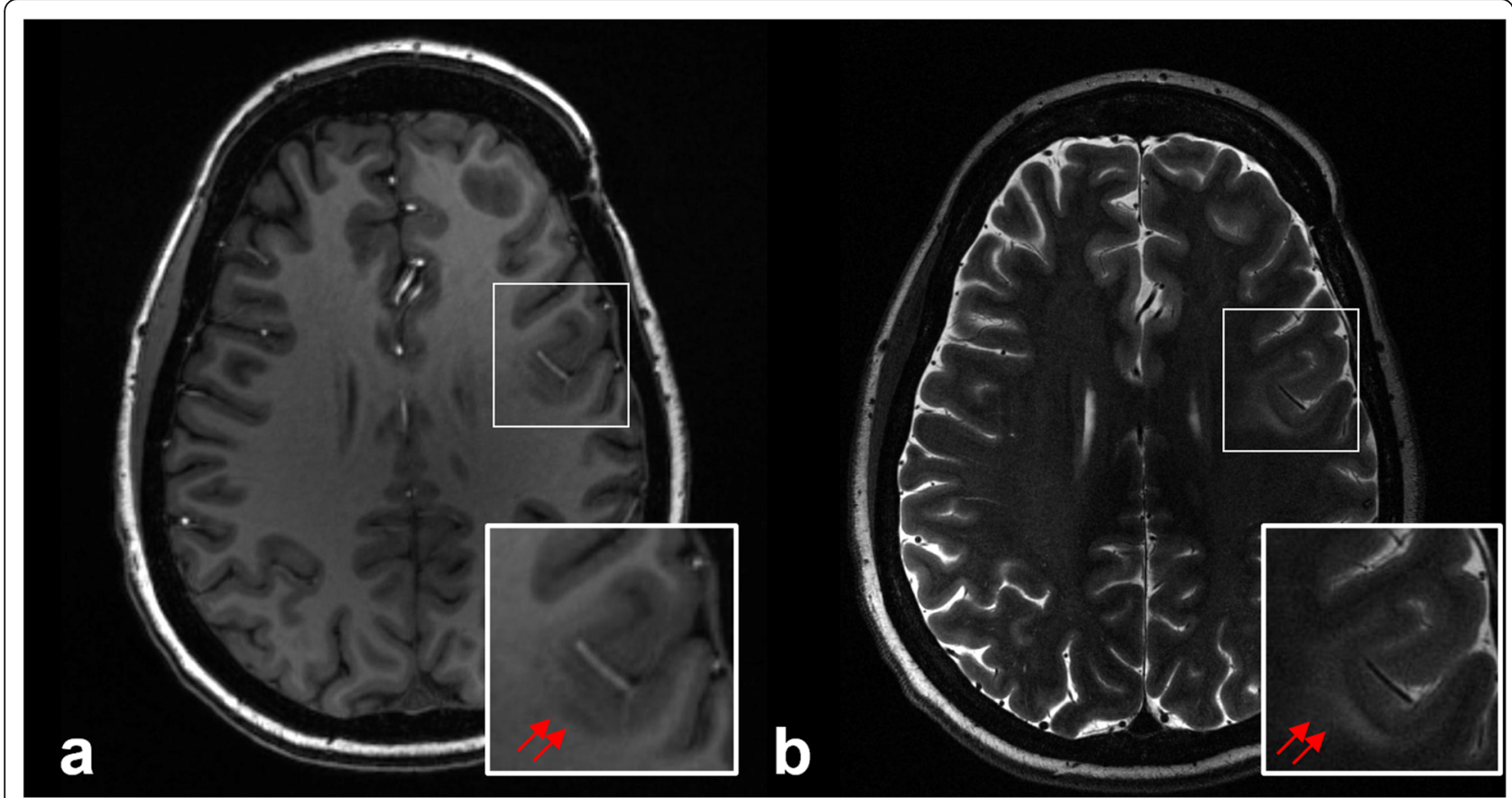

Fig. 2 Anatomical delineation of focal cortical dysplasia at 7 T. Axial T1-weighted (a) and T2-weighted (b) images acquired at 7 T demonstrate blurring of the gray-white matter junction (double arrows), abnormally thick malformed cortex, and an indistinct cortical ribbon in the left inferior frontal gyrus, consistent with focal cortical dysplasia. There is T2 hyperintensity in the subjacent white matter reflecting the white matter component of the dysplasia

the epilepsy patients, the asymmetry was greater in the same lobe as the suspected seizure onset zone. The authors suggested that the quantification of perivascular spaces at $7 \mathrm{~T}$ could provide an important imaging biomarker to study the effects of epilepsy on the brain and assist in localizing the seizure onset zone in surgical planning [27].

Several studies have demonstrated the excellent delineation of hippocampal morphology and internal structures at $7 \mathrm{~T}$. For example, Feldman et al. found improved detection of hippocampal abnormalities in 18 patients at $7 \mathrm{~T}$, and the abnormality was concordant with electroencephalography and clinical findings in 12 of the 18 patients [24]. Similarly, another study was able to demonstrate pathology in cortical area (CA) subfields using ultrahigh field MRI in a small cohort of patients with temporal lobe epilepsy that correlated with histopathological findings [28]. An evaluation of hippocampal internal structure revealed asymmetry in hippocampal vessel density both in neocortical and mesial temporal lobe epilepsy compared to healthy controls, with asymmetrically decreased vessel wall density ipsilateral to the suspected seizure onset zone [29]. Volumetric analyses and magnetic resonance spectroscopy at $7 \mathrm{~T}$ identified hippocampal subfield atrophy commonly affecting CA3 and altered metabolite concentrations (mainly reduced glutamine levels) in the hippocampus of patients with temporal lobe epilepsy [30]. Although the volume and metabolite deviations did not consistently lateralize the epileptogenic hippocampus, lower subiculum volumes and glutamine concentrations correlated with impaired verbal memory performance [30]. Using resting state fMRI at $7 \mathrm{~T}$, Shah et al. [31] demonstrated functional network asymmetry within the mesial temporal lobe that was able to distinguish between temporal lobe epilepsy subtypes, suggesting that the improved SNR and resolution at $7 \mathrm{~T}$ has the potential to improve localization of underlying brain network disruptions in temporal lobe epilepsy patients who are candidates for surgical resection.

In summary, the higher SNR and increased spatial resolution afforded by 7 -T MRI has been shown to improve the sensitivity of detecting epileptogenic lesions and may aid in the identification of morphological variations associated with suspected seizure onset zones.

\section{Multiple sclerosis}

The increased sensitivity of lesion detection and better characterization of lesion pathology at $7 \mathrm{~T}$ promises to provide better differentiation of multiple sclerosis (MS) from other disorders [32]. The identification of a central vein inside white matter lesions on MRI, the central vein sign (CVS), has been proposed as an imaging biomarker in the diagnosis of MS, with many studies suggesting 


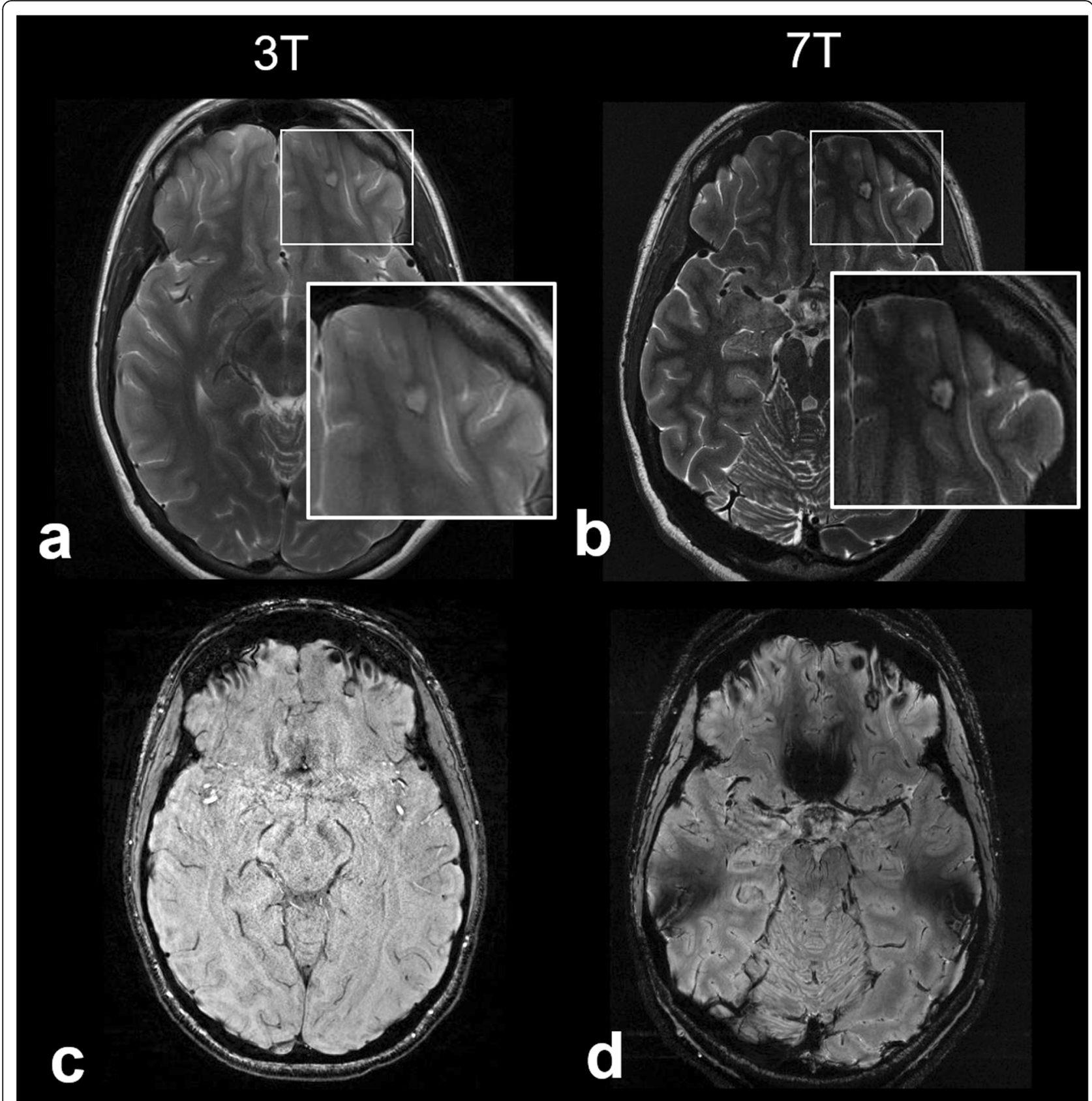

Fig. 3 Cavernous malformation on 3-T and 7-T magnetic resonance imaging. Axial T2-weighted images acquired at $3 \mathrm{~T}(\mathbf{a})$ and $7 \mathrm{~T}$ (b) show a 6$\mathrm{mm}$ cortical lesion in the left orbitofrontal gyrus with central T2 hyperintensity and peripheral T2 hypointensity. Axial susceptibility-weighted images acquired at $3 \mathrm{~T}$ (c) and $7 \mathrm{~T}$ (d) show marked susceptibility effect within the rim of the lesion. The 7-T images have higher signal-to-noise ratio and better delineate the lesion compared to the 3-T images. The lesion was resected and found to be a cavernous malformation on histopathology

that the presence of the CVS accurately differentiates MS from its mimics [33, 34]. In a study of 7 patients with MS and a total of 358 white matter lesions, Tallantyre et al. [35] showed that the CVS was identified in $87 \%$ of lesions at $7 \mathrm{~T}$ compared to $45 \%$ of visible lesions at $3 \mathrm{~T}$ using $\mathrm{T} 2 \%$-weighted sequences. A subsequent study by the same group demonstrated that at $7 \mathrm{~T}, 80 \%$ of lesions in MS patients were perivenous compared to $19 \%$ of lesions in non-MS patients, and that 7-T T2\%weighted MRI reliably distinguished patients with clinically definite MS from those without clinical MS based on the percentage of lesions with the CVS [36]. A study using SWI and magnetization-prepared fluid attenuated inversion-recovery (FLAIR) images at $7 \mathrm{~T}$ demonstrated 


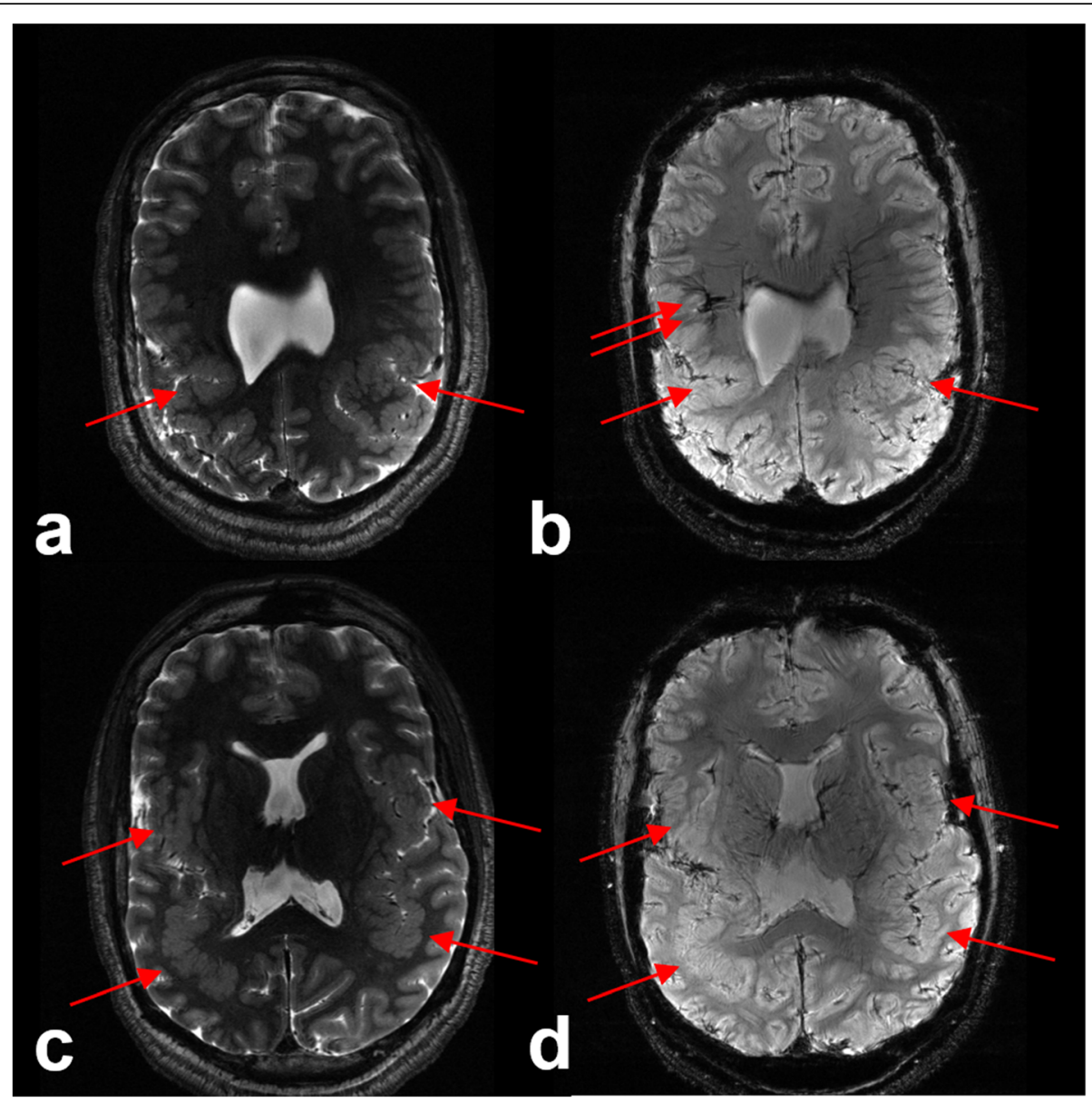

Fig. 4 Sensitivity of 7T magnetic resonance imaging for polymicrogyria. Axial T2-weighted and susceptibility-weighted images at the level of the centrum semiovale $(\mathbf{a}, \mathbf{b})$ and lateral ventricles $(\mathbf{c}, \mathbf{d})$ demonstrate simplification of the gyral folding pattern involving the perisylvian cortex bilaterally, including the posterior temporal lobes and parietal lobes (red arrows), with thickening of the cortex and nodularity, consistent with polymicrogyria. There is also an abnormally large draining vessel on the right (b, red arrow), which may represent a developmental venous anomaly

that at a threshold of $67 \%$, perivenous nonconfluent white matter lesions of $3 \mathrm{~mm}$ in length were able to differentiate relapsing remitting MS patients from healthy controls with a sensitivity of $94 \%$ and a specificity of $100 \%$ [37]. Despite these findings, recent meta-analyses have suggested that while the higher SNR and contrastto-noise ratio of 7-T scanners result in slightly better delineation and detection of the CVS (pooled proportion of central veins detected at $7 \mathrm{~T}=0.82$ ), scanning at $3 \mathrm{~T}$ might be sufficient in this regard (pooled proportion of central veins detected $=0.74$ ), while the use of $1.5-\mathrm{T}$ scanners showed a statistically significant reduction in CVS detection (pooled proportion of central veins detected $=0.58)[38]$.

Beyond the detection of the CVS, the increased spatial resolution at $7 \mathrm{~T}$ has enabled the detection of cortical gray matter lesions in MS, which are frequently observed on histopathological examination but are difficult to detect at lower field strengths due to diminished contrast and lower spatial resolution (Fig. 5) [39-42]. Cocozza et al. [40] demonstrated that cortical lesions can be identified at $7 \mathrm{~T}$ in MS using a three-dimensional (3D)-T1weighted volume that was acquired in a clinically feasible time and that was comparable in performance to that achieved using a T2*-weighted sequence. In a longitudinal study using a quantitative approach that combined T2*-weighted gradient-echo acquisitions at $7 \mathrm{~T}$ with a surface-based analysis, Treaba et al. [42] demonstrated that cortical lesions in MS preferentially developed intracortically and within the cerebral sulci, and that their accumulation was overall independent of white matter lesion accrual, suggesting that the pathogenesis of these lesions are driven by inflammatory cerebrospinal fluid (CSF)-related processes. The authors also reported that cortical lesion accumulation independently predicted neurologic disability progression [42].

The contribution of 7-T MRI for detecting MS white matter lesions is less robust than that for the cortex [43] 


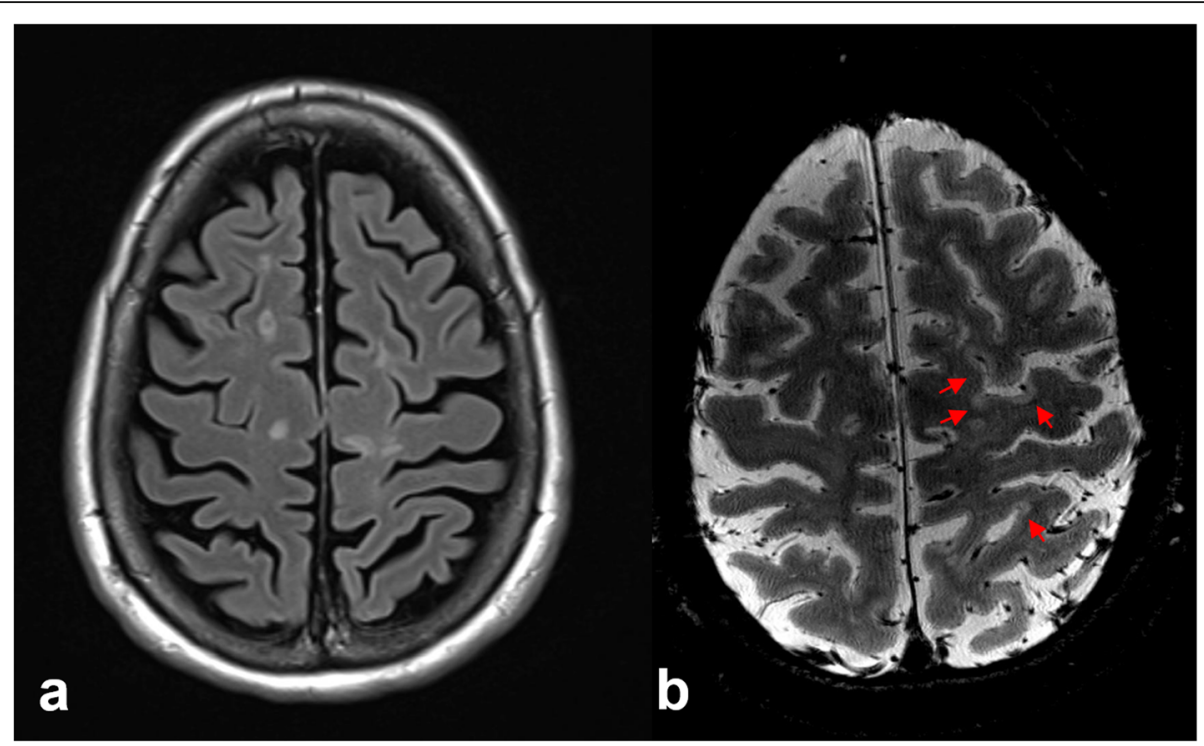

Fig. 5 Cortical lesions in multiple sclerosis on 3-T and 7-T magnetic resonance imaging. Axial fluid-attenuated inversion-recovery (FLAIR) image acquired at $3 \mathrm{~T}(\mathbf{a})$ and axial T2*-weighted image acquired at $7 \mathrm{~T}(\mathbf{b})$. The 7-T image shows many more cortical lesions (red arrows), which are not visible on the axial FLAIR (a) or T2-weighted image (not shown) at $3 \mathrm{~T}$, due to a combination of increased contrast and spatial resolution at $7 \mathrm{~T}$

with ongoing research in the optimization of sequences to reduce limitations secondary to high SAR and to reduce RF field inhomogeneities [43-45]. T2*, FLAIR, magnetization transfer imaging, and magnetizationprepared rapid gradient-echo have been suggested as currently recommended sequences to visualize white matter lesions at $7 \mathrm{~T}[43,46,47]$.

The increased spatial resolution and high SNR afforded by 7-T MRI could also be beneficial in imaging demyelinating lesions of the spinal cord, which remains a challenge at lower field strengths. For example, a small study that compared spinal cord imaging of MS patients at $7 \mathrm{~T}$ versus $3 \mathrm{~T}$ demonstrated better visualization of spinal cord anatomy and 50\% improvement in detection of MS spinal cord lesions at $7 \mathrm{~T}$ compared to $3 \mathrm{~T}$ [48]. Ouellette et al. [49] used 7-T MRI to characterize gray and white matter pathology in the cervical spinal cord of patients with early relapsing-remitting and secondary progressive MS and reported that spinal cord lesions were localized nearest to the subpial surfaces for those with relapsing-remitting MS, whereas lesions in progressive disease were located near the CSF surface of the central canal. This observation lends support to CSFmediated pathogenic mechanisms in lesion development that may differ between MS subtypes. Despite the potential theoretical benefits of imaging the spinal cord in MS at ultrahigh field, technical challenges remain, including the lack of dedicated RF coils that may compromise image quality; small variations in tissue susceptibility that are enhanced at $7 \mathrm{~T}$; and motion artifacts due to respiration, cardiac movement, swallowing and CSF flow $[32,43]$.
In summary, even though the use of ultrahigh field MRI is not yet part of standard-of-care imaging protocols in MS, it has allowed for improved imaging characterization and identification of the different aspects of MS pathophysiology, thereby offering new insights into inter-individual variability and disease evolution.

\section{Brain tumors}

Several studies suggest that 7-T MRI can provide superior information regarding microvascularity and necrosis than 1.5-T and 3-T MRI and could facilitate early stratification of patients with gliomas (Fig. 6), in accordance with recent World Health Organization grading schemes [50-53]. For example, Moenninghoff et al. [51] demonstrated higher tumor microvascularity in high-grade gliomas compared with low-grade lesions using 7-T SWI. These results concurred with those of Grabner et al. [54] who found that a local image variance technique for quantification of hypointense microvascular SWI structures at $7 \mathrm{~T}$ was able to differentiate diffuse infiltrative gliomas based on tumor grade and isocitrate dehydrogenase (IDH) mutational status. Christofordis et al. [55] demonstrated that tumoral pseudoblush obtained using gradient-echo at ultrahigh field correlated histologically with increased microvascularity and overall tumor grade.

TOF MR angiography at $7 \mathrm{~T}$ has recently been used to characterize intratumoral vessels successfully in a small sample of 12 patients with gliomas [56]. The advantages of TOF angiography at $7 \mathrm{~T}$ compared to lower field strengths (increased SNR, longer T1 relaxation times augmenting vessel-tissue contrast and hyperintense 


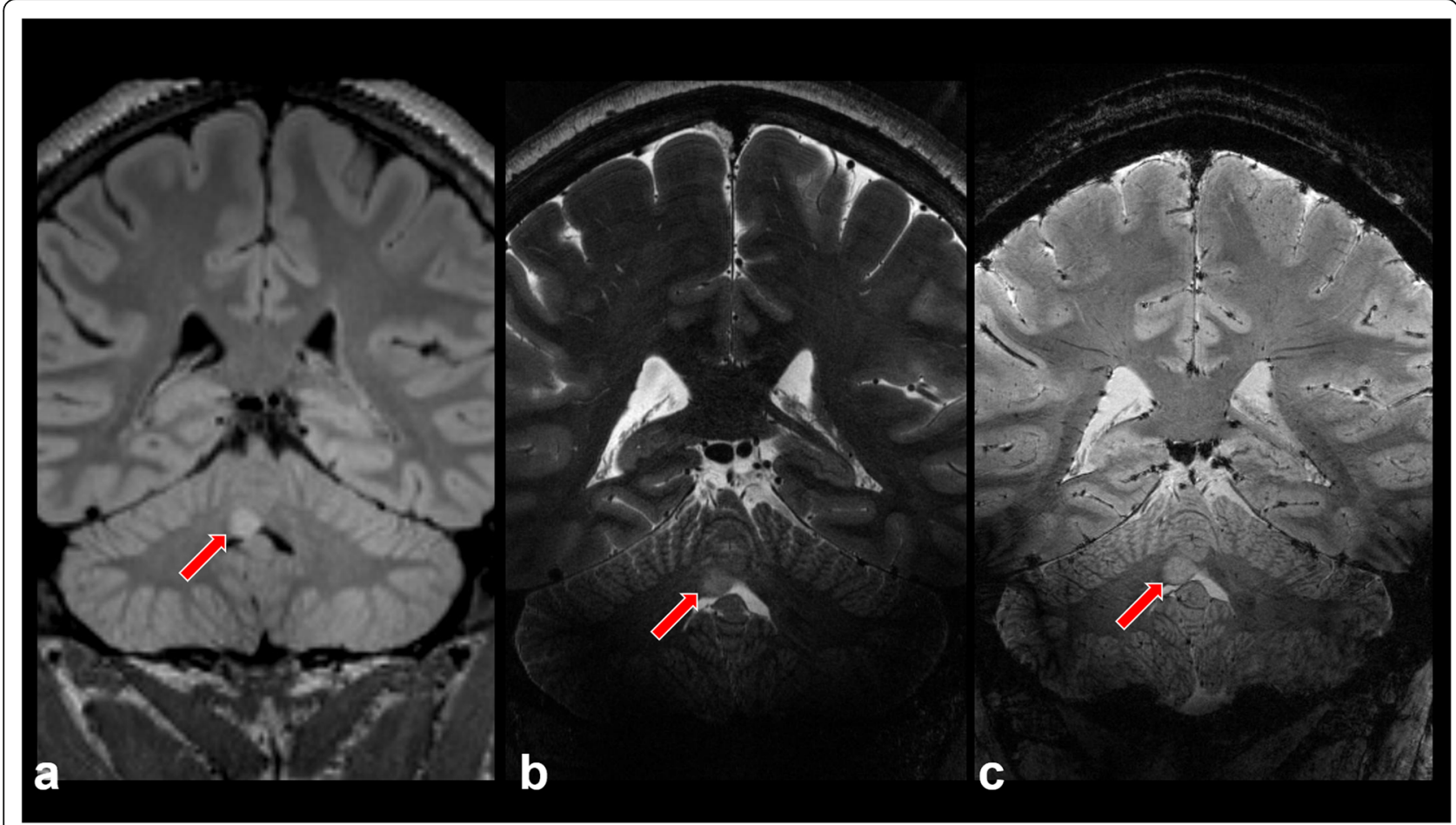

Fig. 6 Hamartoma identified on 3-T magnetic resonance imaging and further characterized at 7 T. Coronal T2-weighted fluid-attenuated inversion-recovery image at $3 \mathrm{~T}$ (a) demonstrates a hyperintense mass (red arrows) in the right cerebellar vermis. Coronal T2-weighted (b) and coronal susceptibility-weighted image (c) obtained at 7T show no evidence of microvascularity within the lesion

arterial vasculature) suggest it may be a useful noninvasive method of grading gliomas since higher grade gliomas tend to display increased angiogenesis. Furthermore, 7-T vascular imaging has been leveraged to study longitudinal glioma microvasculature changes after antiangiogenic therapy, with a potential role in the noninvasive monitoring of antiangiogenic therapy response in patients with aggressive brain tumors [57].

High-resolution FLAIR images at $7 \mathrm{~T}$ were compared with clinical FLAIR sequences at $3 \mathrm{~T}$ in patients with glioblastoma prior to radiotherapy planning and yielded significantly higher SNR in white matter and better contrast between gray and white matter compared to clinical FLAIR sequences [58]. The authors suggested that the better visibility of anatomical borders afforded by improved gray matter and white matter contrast at 7-T FLAIR could be leveraged to improve the delineation of brain structures at risk during treatment planning. A recent study demonstrated that sodium $\left({ }^{23} \mathrm{Na}\right)$ MRI at $7 \mathrm{~T}$ correlated with isocitrate dehydrogenase (IDH) mutation status in glioma patients with potential application in image-guided biopsy, surgery, and radiotherapy [59]. The authors also reported a successive decrease of ${ }^{23} \mathrm{Na}$ concentration from regions of central necrosis to normal appearing white matter suggesting a correlation with tumor infiltration.
Magnetic resonance spectroscopy (MRS) at higher field strengths improves SNR and spectral dispersion, leading to improved sensitivity and specificity, respectively, to different chemical species and metabolites. MRS has the potential to improve the detection of metabolites for tumor characterization; however, the technical challenges of increased SAR, magnetic field inhomogeneities, and fast $\mathrm{T} 2 \%$-relaxation can impose constraints on the acquisition of MRS data at $7 \mathrm{~T}$ [60-62]. Despite these limitations, earlier research at $7 \mathrm{~T}$ was able to demonstrate the application of a short-echo spin-echo MRS sequence to detect characteristic differences in regions of tumor versus normal appearing brain and successfully discriminate between short-echo metabolites, particularly glutamate and glutamine, although with low spatial resolutio $\mathrm{n}[63]$; this differentiation remains elusive at field strengths of $3 \mathrm{~T}$ and below [60]. The last decade has seen different MRS acquisition approaches to offset some of the challenges at ultrahigh field imaging [60, 64-67]. For example, Hangel et al. [66] used 7-T MRS combined with patch-based super-resolution (PBSR) reconstruction in ten glioma patients that resulted in the identification of complex metabolic activities, which were in topographic agreement with tracer uptake on PET. In a subsequent study, Hangel et al. [60] successfully demonstrated metabolic differences between tumor regions and peritumoral tissues for multiple metabolites 
using a fast high-resolution whole-brain 3D MRS method in 23 patients with histologically verified high grade gliomas. Although 2-hydroxyglutarate (2HG) was not satisfactorily quantified in this study, other studies have demonstrated detection of $2 \mathrm{HG}$ at ultrahigh field [64, 68, 69]. For example, using a dual-read out alternated gradients echo-planar spectroscopic imaging (DRAG-EPSI) approach at $7 \mathrm{~T}$, An et. al. demonstrated reliable high-resolution $2 \mathrm{HG}$ imaging in a small cohort of glioma patients [64]. Bisdas et al. demonstrated 2HG using MRS at 9.4 T [69]. However, additional validation studies using larger sample sizes are needed for quantification of 2HG and other cancer metabolites at ultrahigh field.

Chemical exchange saturation transfer imaging (CEST), a novel contrast technique that can detect low concentration metabolites with higher sensitivity than MRS, has been used both at clinical and ultrahigh field strengths to differentiate gliomas [70-74]. Paech et al. [73] demonstrated that relaxation-compensated amide proton transfer (APT) MRI was significantly correlated with patient overall survival and progression-free survival in newly diagnosed high-grade glioma patients. CEST-derived contrasts at $7 \mathrm{~T}$, particularly Nuclear Overhauser effect (NOE) imaging as well as downfield-NOE-suppressed APT, were demonstrated to be significant predictors of early progression after first-line therapy in twenty previously untreated glioblastoma patients [75]. Meissner et al. demonstrated that relaxationcompensated relayed NOE (rNOE)-mediated CEST imaging at $7 \mathrm{~T}$ was able to discriminate responders from nonresponders immediately after the end of chemoradiotherapy in 12 patients with gliomas which was at least 4 weeks earlier than the standard clinical evaluation by Response Assessment in Neuro-Oncology, suggesting that CEST MRI has the potential to enable early response assessment in glioma patients [72]. Paech et al. [74] demonstrated that relaxation-compensated multipool CEST MRI, particularly downfield-rNOE-suppressed-APT imaging, enabled the prediction of IDH mutation status and differentiation of high-grade from low-grade gliomas, suggesting the potential to use this technique as a noninvasive imaging biomarker in the diagnostic workup of these tumors. A recent study showed that CEST signal intensities at $7 \mathrm{~T}$ are dependent on the anatomic location of high-grade gliomas such that NOE showed significant dependence on subventricular zone contact while amide CEST signals depended on the hemispheric location of the glioma [70]. These recent findings, along with associated contaminants and the occasional difficulty in how to interpret these signals, suggest the need for additional investigation before the adoption of these techniques into routine clinical practice [53].

In summary, ultrahigh field MRI improves the detection of small metastases, delineation of tumor extent, characterization of the intratumoral microenvironment, and detection of post-treatment effects when compared to conventional strength MRI. However, technical challenges related to MRS and novel CEST techniques remain and warrant additional research.

\section{Neurodegenerative disorders}

Studies at $7 \mathrm{~T}$ within the field of neurodegenerative disorders have focused mainly on (1) dementia including Alzheimer's disease (AD) and vascular dementia and (2) Parkinson's disease (PD). Although hyperphosphorylated tau and amyloid beta are considered key mediators in the pathogenesis of $\mathrm{AD}$, an increasing number of studies suggest that altered iron metabolism also plays an important role [76-80]. T2*-weighted MRI at $7 \mathrm{~T}$ performed on postmortem frontal cortex of patients with early-onset and late-onset AD as well as controls demonstrated severe disruption of the cortical lamination in $\mathrm{AD}$ patients, which correlated with changes in cortical myelin and iron accumulation [76]. A subsequent study by the same group evaluated postmortem medial temporal lobe in $\mathrm{AD}$ and controls and demonstrated more variety in visible cortical lamination in $\mathrm{AD}$ patients as well as severely distorted cortical lamination in advanced stage AD patients; the changes in appearance of visible cortical lamination were associated with diffuse cortical iron alterations and iron deposition [78].

A series of studies have used 7-T MRI to delineate the pattern of atrophy of different substructures of the hippocampus in $\mathrm{AD}$ with implications for understanding the neuropathogenesis involved in memory loss in these patients [81-84]. Bouvy et al. [85] demonstrated a topographical association between a high degree of juxtacortical perivascular space dilation adjacent to cortical cerebral microbleeds in amnestic mild cognitive impairment and early AD patients that may be due to impaired drainage of interstitial fluid related to perivascular amyloid deposition. The authors suggested that dilated perivascular spaces at ultrahigh field may act as an indirect neuroimaging marker for cerebral amyloid angiopathy in AD.

Deep brain stimulation (DBS) of target nuclei such as the subthalamic nucleus, globus pallidus pars interna, and ventralis intermedius nucleus of the thalamus is a widely performed surgical treatment for patients with $\mathrm{PD}$ in which an electrode is placed to stimulate the motor components while avoiding the associative and limbic components [86]. Improved imaging contrast, resolution, and SNR at $7 \mathrm{~T}$ afford improved visualization of DBS target nuclei compared to standard 1.5-T or 3-T scanners [87-91]. Patriat et al. [89] used tractographybased parcellation at $7 \mathrm{~T}$ in PD patients prior to DBS surgery to segment the globus pallidus pars interna into motor, associative, and limbic components and identified functional territories that were organized in a reproducible manner. Similarly, using a structural connectivity- 
based parcellation protocol, the subthalamic nucleus' connections to the motor, limbic, and associative cortical areas were used to map the individual subcomponents of the nucleus in patients with idiopathic PD [90], further reinforcing the role of ultrahigh field imaging in facilitating individualized planning of deep brain stimulation surgery.

Differences in anatomical patterns of the substantia nigra between PD patients and healthy volunteers using 7-T MRI have also been described [92], which could allow for an earlier diagnosis of the disease. A recent study demonstrated significant changes in subthalamic nucleus morphology (lower volume) and microstructural organization (lower fractional anisotropy) in patients with mild-moderate PD compared with healthy older adults, suggesting that neurodegenerative changes in PD extend beyond the substantia nigra pars compacta to involve the hyperdirect and indirect basal ganglia pathways [93]. Poston et al. leveraged quantitative susceptibility mapping at $7 \mathrm{~T}$ to segment individual midbrain nuclei that are associated with PD progression in 32 participants with PD [94]. The authors reported that smaller substantia nigra volumes in mild-moderate PD correlated with longer disease duration and more severe bradykinesia-rigidity scores, but not to tremor or postural instability scores. Finally, ultrahigh field imaging has been used to identify changes in hippocampal subfields in patients with $\mathrm{PD}$, adding to our understanding of the underlying neuroanatomical mechanisms of episodic memory impairments seen in PD [95].

In summary, increased image resolution at $7 \mathrm{~T}$ MRI enables the visualization of small structures of interest in neurodegenerative pathophysiology including cortical layers, hippocampal subfields, brainstem nuclei, plaques, and microbleeds. 7-T MRI has been successfully employed to detect biomarkers of disease in both $\mathrm{AD}$ and $\mathrm{PD}$ and may offer a powerful complement to other noninvasive imaging modalities in the evaluation of dementia.

\section{Cerebrovascular diseases}

MRI remains the most sensitive imaging technique to detect acute infarction. Clinically feasible stroke imaging protocols have been designed to image subacute and chronic infarcts at $7 \mathrm{~T}$ [96]. The exclusion of diffusionweighted imaging (DWI) as part of the protocol precludes its use in current clinical practice for the evaluation of acute stroke patients [97]. Despite this, the high SNR, high spatial resolution, and high CNR afforded by ultrahigh field MRI can be used to evaluate small cerebrovascular lesions (such as cortical microinfarcts) and vasculature on the submillimeter scale, thereby allowing better characterization of cerebrovascular disease including stroke using $7 \mathrm{~T}$ compared to lower field strengths $[52,97,98]$.
MR angiography at $7 \mathrm{~T}$ has been used successfully to image occlusive changes in small vessels such as the lenticulostriate arteries in acute and chronic strokes; these vessels are difficult to image with conventional modalities $[99,100]$. Further, when images of patients with subacute and chronic strokes were compared at $7 \mathrm{~T}$ and $3 \mathrm{~T}$, the higher spatial resolution of 7-T scanners revealed subtle features of infarct morphology compared to the lower field MR images [96].

Intracranial atherosclerosis is a major risk factor of ischemic stroke [101]. Beyond depicting the lumen of smaller caliber intracranial arteries, research has leveraged the advantages of ultrahigh field imaging to evaluate directly the intracranial vessel wall [102-104]. MRI at $7 \mathrm{~T}$ was superior to $3-\mathrm{T}$ MRI in vessel wall characterization and visualization of the fibrous cap and lipid core within atherosclerotic plaques in patients with intracranial atherosclerotic disease [105], suggesting the potential role of 7-T MR in risk stratification in intracranial atherosclerosis. Another study correlating in vitro intravascular sonography and 7-T MRI findings with histology concluded that 7-T MRI is a reliable method for detecting atherosclerotic burden within the intracranial arteries [106]. A recent study in 130 patients with a history of vascular diseases examined the association between intracranial atherosclerosis burden measured with intracranial vessel wall sequences at $7 \mathrm{~T}$ and several markers of extracranial atherosclerosis and found that ankle brachial index, the presence of extracranial carotid stenosis, carotid intima-media thickness, and decreasing glomerular filtration rate were all associated with a higher intracranial atherosclerotic burden, suggesting that similar atherogenic mechanisms underlie both processes [104]. Lindenholz et al. [102] investigated the association between intracranial vessel wall lesion burden and vascular risk factors in patients with ischemic stroke or transient ischemic attack of the anterior circulation. The authors reported that other than smoking, established common risk factors such as increasing age, diabetes, and hypertension were associated with a higher number and greater enhancement of intracranial vessel wall lesions in these patients. The same group used 7-T MRI to demonstrate differences in the appearance of the vessel wall in patients who underwent thrombosuction treatment after ischemic stroke compared to patients with stroke who did not undergo thrombectomy. The clinician should be aware of these differences to avoid misinterpretation on post-treatment follow-up imaging [103].

The increased SNR, higher spatial resolution, and better fluid suppression seen at $7 \mathrm{~T}$ have been leveraged to evaluate patients with aneurysms that are at high risk for rupture, predict potential for atherosclerotic plaque rupture, and characterize infarct morphology [52]. For example, Sato et al. used gadolinium-enhanced T1- 
weighted magnetization prepared rapid gradient recalled echo (MPRAGE) sequences at $7 \mathrm{~T}$ to describe aneurysm wall microstructures responsible for gadolinium enhancement not seen at lower field strengths. Partial or complete inner wall enhancement correlated with neovascularization of the inner wall layer as well as the adjacent thrombus, and partial or complete outer wall enhancement could be explained by formation of vasa vasorum in the outer aneurysm wall layer [107]. The authors concluded that the double-rim enhancement seen at $7 \mathrm{~T}$ correlated with perifocal edema and wall histologic findings suggestive of instability. In another study that compared 7 $\mathrm{T}$ and $1.5 \mathrm{~T}$, the detection and characterization of unruptured aneurysms was demonstrated to be better at $7 \mathrm{~T}$ [108]. Wrede et al. [109] demonstrated that 7-T TOF MR images were comparable to digital subtraction angiography, the current gold standard.

In summary, ultrahigh field vessel wall imaging in the evaluation of cerebrovascular disease may enable earlier diagnosis, better differentiation of the underlying disease processes, and provide valuable information in the management intracranial vascular disease. However, the exclusion of DWI limits use of 7-T MRI in the evaluation of acute stroke patients at this time.

\section{Ultra-high and ultrafast gradient technology}

\section{Technological overview and state-of-the-art}

As a major component of the scanner, the gradient system plays a key role in MR image generation by enabling spatial selection and image encoding. The performance characteristics of the gradient system are parameterized by the maximum gradient amplitude, which is measured in $\mathrm{mT} / \mathrm{m}$, and the slew rate, which describes how fast a gradient can attain a desired amplitude within a given amount of time $(\mathrm{T} / \mathrm{m} / \mathrm{s})$. The magnetic field gradients have tremendous influence on the overall quality of the acquired image, a point that has gained increasing focus from the major scanner manufacturers in the quest for improved image quality and ever-faster image encoding speed. Considerable engineering efforts have been made in the last 30 years to maximize the performance of gradient systems for clinical use. The most recent generation of commercial scanners such as the Siemens Prisma, GE Premier, and Philips Achieva 3T X-series (Quasar Dual gradient system) feature integrated wholebody gradient systems with a maximum gradient amplitude of $80 \mathrm{mT} / \mathrm{m}$ and maximum slew rates of 200, 200, and $100 \mathrm{~T} / \mathrm{m} / \mathrm{s}$, respectively.

For applications such as diffusion MRI, gradient coils capable of producing gradient fields with high amplitudes significantly reduce the time required for diffusion encoding and improve the SNR of such measurements by reducing signal decay from T2 relaxation. Diffusion MRI has been the driving motivation behind recent efforts to engineer dedicated gradient coils with large maximum gradient amplitudes. For example, customized gradient coil inserts such as the Siemens SC72 gradient insert that was designed for the Washington UniversityMinnesota Consortium Human Connectome Project scanner are capable of generating maximum gradient strengths of $100 \mathrm{mT} / \mathrm{m}$ [110]. The Connectom wholebody gradient coil designed for the Massachusetts General Hospital-University of Southern California Human Connectome Project utilized a segmented design with 12 total gradient amplifiers (four 900 A drivers at $2250 \mathrm{~V}$ each per physical axis) to overcome efficiency limits and attain a maximum gradient amplitude of $300 \mathrm{mT} / \mathrm{m}$ and maximum slew rate of $200 \mathrm{~T} / \mathrm{m} / \mathrm{s}$ within a $56-\mathrm{cm}$ bore diameter [111]. Smaller diameter head gradient coils are a viable alternative to achieving even higher maximum gradient amplitudes compared to whole-body gradient coils and have remained a design of interest for integration into 3-T MRI systems using clinically available gradient power amplifiers. As an example, the MAGNUS (Microstructure Anatomy Gradient for Neuroimaging with Ultrafast Scanning) head gradient coil offers 200 $\mathrm{mT} / \mathrm{m}$ maximum gradient amplitude and slew rate of up to $500 \mathrm{~T} / \mathrm{m} / \mathrm{s}$ per axis within a $37-\mathrm{cm}$ bore diameter using lower power amplifiers $(620 \mathrm{~A} / 1,500 \mathrm{~V})$ than those used in the Connectom scanner [112].

\section{Challenges and opportunities of imaging with higher performance gradient systems}

Higher performance gradient systems equipped with higher maximum gradient amplitudes and slew rates enable increased spatial resolution and faster acquisitions. To achieve higher spatial resolution, larger gradient areas are required, noting that the maximum area under a gradient encoding pulse determines the highest achievable spatial resolution. This can be achieved using lower gradient strengths by lengthening the gradient encoding pulses without increasing their amplitudes; unfortunately, this approach leads to a loss of SNR due to T2related signal decay during the image readout period. To shorten the image readout time without sacrificing resolution, the gradient amplitude must increase, and the time to achieve the desired gradient amplitude must be minimized. High-performance gradient systems are thus designed to maximize gradient amplitude and slew rate within technical and biological limits. Shortening the gradient pulses during image readout must be carefully balanced against decreasing the SNR of the image beyond an acceptable limit, as the SNR is inversely related to the square root of the bandwidth, which is set by the gradient amplitude.

Technical limitations include the engineering challenges of achieving a spatially linear magnetic gradient field, removal of heat from the gradient coil, and 
efficiency and power requirements of the coil. While these technical challenges have largely been addressed in the modern era, including advances in gradient and shim technology [113-115], the main limiting factor on gradient coil performance is the biological effect of rapidly switching, large magnetic fields on the human body. Peripheral nerve stimulation (PNS) can be induced if the product of the gradient switching rate and strength generate electric fields large enough to trigger nerve firing, which can be uncomfortable and potentially painful. Due to such safety concerns, peripheral nerve stimulation is strictly regulated in MRI. The incorporation of anatomical models of the peripheral nerves into electromagnetic and neurodynamic simulations of PNS [116] promises to enable more accurate prediction of PNS thresholds and may inform the design of gradient coils to minimize PNS [117]. Smaller head gradients covering only the head and neck regions eliminate the induction of current paths in the chest and back, which are particularly prone to stimulation, and offer another strategy for reducing PNS in brain imaging [118].

\section{Clinical applications of ultrahigh gradient technology Anatomic MRI}

Stronger and faster gradients benefit anatomical imaging by enabling more efficient image readout, with a concomitant reduction in echo time and repetition time, depending on the image contrast of interest. The shortened acquisition time can then be used to increase spatial resolution and/or SNR through signal averaging within a similar overall scan time. For example, using the Connectome whole-body gradient system with a maximum gradient strength of $300 \mathrm{mT} / \mathrm{m}$ and slew rate of $200 \mathrm{~T} / \mathrm{m} / \mathrm{s}$, and comparing against a whole-body gradient system with $60 \mathrm{mT} / \mathrm{m}$ and $200 \mathrm{~T} / \mathrm{m} / \mathrm{s}$ maximum gradient strength and slew rate, improvements in anatomical imaging include $\sim 25 \%$ reduction in the repetition time and $\sim 50 \%$ reduction in echo time for the $3 \mathrm{D}$ T1-weighted MPRAGE sequence due to shorter echo spacing, which translates into a roughly $\sim 25 \%$ reduction in acquisition time.

The constant push for shorter scan times has led to the development of more efficient k-space sampling schemes for 2D and 3D imaging, which benefit from better gradient performance. Spiral k-space encoding makes efficient use of the gradient system hardware [119] and has been used to achieve ultrashort echo times for realtime and rapid imaging applications [120]. As another example, wave-controlled aliasing in parallel imaging (CAIPI) is a 3D parallel imaging technique that uses a controlled pattern of gradient modulation in the phaseand partition-encoding directions to create 2D CAIPI shifts between partitions and voxel spreading in all three dimensions using sinusoidal gradients $[121,122]$. Wave-
CAIPI takes full advantage of the 3D coil sensitivity information when using high-channel count array coils to provide high acceleration factors with negligible artifacts and g-factor penalty across a variety of contrasts. In general, higher gradient amplitudes and lower bandwidth generate more voxel spreading [123], and higher slew rates allow for an increased number of sinusoidal cycles per encoding period, which diminish the amount of artifact in the resulting images. Wave-CAIPI takes advantage of higher gradient strengths and fast slew rates to enable higher resolution and faster anatomical imaging. The diagnostic performance of Wave-CAIPI has been shown to be equivalent to that of standard 3D anatomical sequences acquired with conventional parallel imaging across a variety of contrasts with reduced scan time and motion artifacts [123-126] (Fig. 7).

\section{Diffusion MRI}

High-performance gradient systems equipped with large maximum gradient amplitudes are particularly beneficial for diffusion MRI as they boost the efficiency of diffusion encoding. The use of stronger gradients enables a given diffusion-encoding gradient area to be achieved in less time and effectively shortens the entire diffusionencoding period and echo time, resulting in less signal loss from T2 relaxation. Beyond the increase in SNR, larger gradient amplitudes enable stronger diffusion encoding to be achieved with shorter effective diffusion times, which improves the sensitivity of the diffusion MRI measurement to microscopic structures on the micron scale [127] and improves the resolution of fine structures such as crossing fibers for diffusion tractography $[128,129]$. The availability of dedicated highperformance gradient coils with large maximum gradient amplitudes enables new classes of diffusion MRI measurements to be performed in the living human brain and provides a more sensitive probe of white matter microstructure in various neurological diseases. High gradient amplitudes benefit the estimation of tissue microstructural properties such as neurite density and orientation dispersion [130], axon diameter [131-135], and the dimensions of the extracellular space [136], which offer greater specificity to tissue microstructure changes than diffusion tensor imaging.

Among the tissue microstructural properties that can be measured using diffusion MRI, axon diameter estimation requires high gradient amplitudes to sensitize the diffusion MRI signal to intra-axonal water diffusion [134, 137-139]. Axon diameter mapping using highgradient diffusion MRI has been applied to study changes in axonal structure and packing density in MS and the aging brain. Axon diameter mapping in the corpus callosum of relapsing-remitting and progressive MS patients has revealed increased axon diameter and 


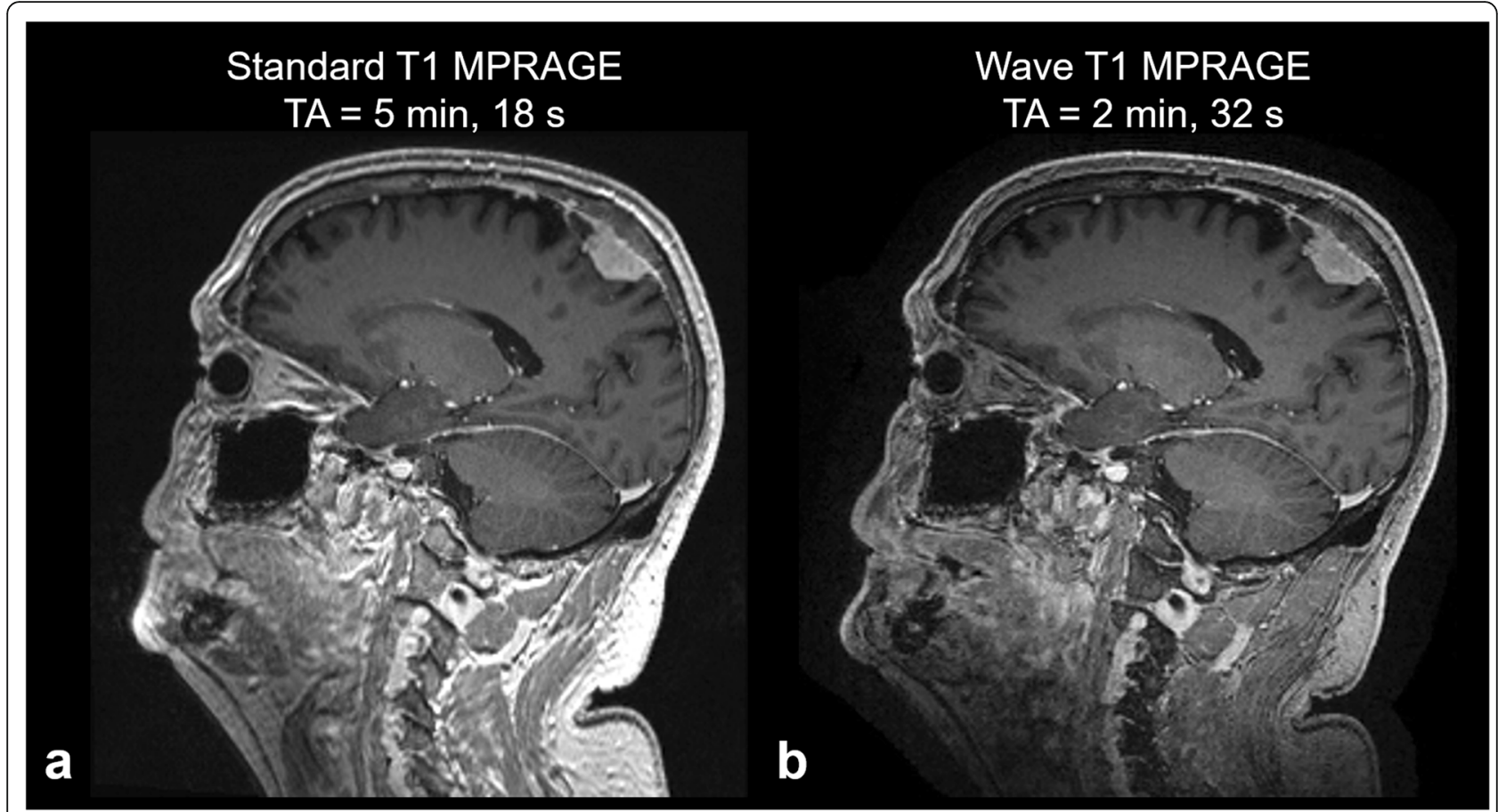

Fig. 7 Comparison of post-contrast three-dimensional T1-weighted magnetization prepared rapid acquisition gradient recalled echo (MPRAGE) images acquired with conventional parallel imaging and wave-controlled aliasing in parallel imaging (CAIPI) encoding demonstrating a meningioma. Standard (a) and Wave-CAIPI (b) T1-weighted MPRAGE images show equivalent visualization of the dural-based enhancing mass along the parietal convexity. Both images were acquired on a 3-T Siemens Prisma MRI scanner equipped with $80 \mathrm{mT} / \mathrm{m}$ maximum gradient strength and $200 \mathrm{~T} / \mathrm{m} / \mathrm{s}$ maximum slew rate. The Wave-CAIPI sequence was more than twice as fast as the standard sequence (acquisition time $=$ $2 \mathrm{~min}, 32 \mathrm{~s}$ for Wave-CAIPI compared to $5 \mathrm{~min}, 18 \mathrm{~s}$ for the standard sequence)

decreased axon density in lesions and the normalappearing white matter [140-142], in keeping with findings on histopathology, which show decreased axon density and an overall increase in axon diameter in postmortem MS tissue [143]. The observed increase in axon diameter as measured by high-gradient diffusion MRI is also a strong predictor of disability and cognitive dysfunction [144], particularly in tests of interhemispheric processing speed and working memory, which rely on the corpus callosum as the major white matter fiber tract mediating these processes. The application of axon diameter mapping to the aging brain has uncovered significant increases in axon diameter with advancing age in the anterior white matter [145], with changes most pronounced in the genu of the corpus callosum and forceps minor. These alterations in axon diameter are consistent with previously reported regional decreases in fractional anisotropy within the frontal white matter [146] and parallel decreases in corpus callosum area and regional gray matter volume with age.

The availability of ultrastrong gradients for diffusion MRI has enabled the translation of novel diffusionencoding paradigms to probe brain tissue microstructure in patients, including oscillating-gradient waveforms
[147], double-diffusion encoding [148], and q-space trajectory imaging [149]. One recent study combined isotropic diffusion encoding with ultrastrong diffusion gradients to achieve high diffusion-weighting in highly restricted, spherical compartments (the so-called dot compartment) in the cerebellar gray matter while suppressing signal arising from anisotropic water within axons [150]. By gaining greater specificity to cellular signatures in the cerebellum, spherical tensor encoding performed with high gradient strengths may enable the earlier identification of cerebellar gray matter loss in patients with hereditary ataxias such as spinocerebellar ataxia type 2, which selectively affects the granule and Purkinje cells. The ability to probe microscopic diffusion anisotropy in brain tumors using q-space trajectory imaging may enable the differentiation of brain tumors such as meningiomas from high-grade glial tumors based on their cellular morphology and composition [151].

The results of clinical research studies demonstrate the potential of high-gradient diffusion MRI to uncover changes in axonal and cellular microstructure and motivate the continued development, application, and dissemination of high-gradient technology for use in commercially available human MRI scanners. 


\section{Functional MRI}

Higher gradient strengths and faster slew rates facilitate the development and application of accelerated fMRI acquisitions at high spatial resolution. Higher gradient strengths and faster slew rates translate directly into better image quality for echo-planar imaging, the workhorse of functional MR image readout, by boosting the SNR, reducing distortions, and enabling higher in-plane resolution and thinner slices. Beyond the benefits to image quality, the fast slew rates available on high-performance gradient systems promise to enhance the acquisition efficiency and dense sampling of $\mathrm{T} 2$ and $\mathrm{T} 2 *$ signal evolution for fast quantitative mapping of the BOLD signal arising from the micro- and microvasculature. Acquiring fMRI data at higher temporal and/or spatial resolution promises to provide new information about neuronal activation, particularly in the early phases of the BOLD response that are thought to reflect the fast response of smaller blood vessels in the brain to local neuronal activity. Traditionally, fMRI has been thought to be too slow to measure brain oscillations due to the dependence of the fMRI signal on slow changes in blood flow. However, recent studies using ultrafast fMRI at 3 and $7 \mathrm{~T}$ have revealed fast neural oscillations at up to $0.75 \mathrm{~Hz}$ in the visual cortex following high-frequency visual stimulation $[152,153]$. Although it remains to be seen whether these signals carry additional information beyond that embedded in the slower BOLD oscillations, the possibility of acquiring such signals promises to uncover abnormal fluctuations in small deep brain structures, such as brainstem nuclei, while correcting for rapid physiological noise (e.g., cardiac and respiratory motion around the brainstem). Given that the pathophysiological hallmark of Parkinson's disease is a degeneration of several neurotransmitter systems in the brain stem, these new techniques may make it possible to test for altered activity and functional connectivity of these nuclei in PD and other neurodegenerative disorders.

\section{Conclusions}

MRI technology development over the last 20 years has focused on pushing the limits of static magnetic field strength and gradient amplitude and speed. Advances in imaging at ultrahigh field, particularly at $7 \mathrm{~T}$, have effectively ushered in next-generation image quality for clinical neuroimaging, with better SNR and higher resolution scans that provide better visualization of small and subtle lesions such as cortical lesions in MS and cortical malformations in epilepsy. At the same time, advances in gradient technology have enabled high-fidelity image acquisition within shorter scan times, and have also enabled better resolution of tissue microstructure through diffusion MRI, allowing for the first time the characterization of microscopic tissue features in the living human brain paralleling what can be seen directly on histopathology. Building on the success of these advances, the next few decades will likely see a continued push toward even higher field strengths and higher gradient strength systems, while encouraging the more widespread adoption of ultrahigh field and ultrahigh, ultrafast gradient technology for clinical use.

\section{Abbreviations \\ 3D: Three-dimensional; AD: Alzheimer's disease; APT: Amide proton transfer; BOLD: Blood oxygenation level dependent; CA: Cortical area; \\ CAIPI: Controlled Aliasing in Parallel Imaging; CEST: Chemical exchange saturation transfer imaging; CVS: Central vein sign; FLAIR: Fluid attenuation inversion-recovery; fMRI: Functional magnetic resonance imaging; IDH: Isocitrate dehydrogenase; MAP: Morphometric analysis program; MPRAGE: Magnetization prepared rapid gradient recalled echo; MR: Magnetic resonance; MRI: Magnetic resonance imaging; MS: Multiple sclerosis; NOE: Nuclear Overhauser effect; PD: Parkinson's disease; PNS: Peripheral nerve stimulation; rNOE: Relayed NOE; SAR: Specific absorption rate; SNR: Signal-to-noise ratio; SWI: Susceptibility-weighted imaging; TOF: Time of flight}

\section{Acknowledgements}

We thank Dr. Augusto Lio Goncalves Filho for sharing the images used in Fig. 7.

\section{Authors' contributions}

SYH and BV conceived the work and wrote the manuscript. SYH prepared the figures. All authors read and approved the final manuscript.

\section{Funding}

SYH. received funding support from the National Institute of Biomedical Imaging and Bioengineering and the National Institute of Neurological Diseases and Stroke of the National Institutes of Health under award numbers: U01EB026996, P41EB03006, R21AG067562, R01NS118187, and K23NS096056. BV received funding support from the National Cancer Institute of the National Institutes of Health under award number P30CA008748

\section{Availability of data and materials}

Not applicable.

\section{Declarations}

Ethics approval and consent to participate

Not applicable.

Consent for publication

Not applicable.

\section{Competing interests}

S.Y.H. received a research grant from Siemens Healthineers for the clinical translation of fast neuroimaging techniques. B.V. declares no competing interests.

\section{Author details}

'Department of Radiology, Memorial Sloan Kettering Cancer Center, 1275 York Ave, New York, NY 10065, USA. ${ }^{2}$ Athinoula A. Martinos Center for Biomedical Imaging, Department of Radiology, Massachusetts General Hospital, 149 13th Street, Room 2301, Charlestown, MA 02129, USA.

Received: 4 November 2020 Accepted: 30 March 2021

Published online: 26 August 2021

\section{References}

1. Robitaille PM, Abduljalil AM, Kangarlu A et al (1998) Human magnetic resonance imaging at 8 T. NMR Biomed 11:263-265 https://doi.org/10.1002/ (sici) 1099-1492(199810)11:6<263::aid-nbm549>3.0.co;2-0

2. Budinger TF, Bird MD (2018) MRI and MRS of the human brain at magnetic fields of 14T to 20T: Technical feasibility, safety, and neuroscience horizons. Neuroimage. 168:509-531 https://doi.org/10.1016/j.neuroimage.2017.01.067 
3. Pohmann R, Speck O, Scheffler K (2016) Signal-to-noise ratio and MR tissue parameters in human brain imaging at 3, 7, and 9.4 tesla using current receive coil arrays. Magn Reson Med 75:801-809 https://doi.org/10.1002/ mrm.25677

4. Tian Q, Bilgic B, Fan Q et al (2020) Improving in vivo human cerebral cortical surface reconstruction using data-driven super-resolution. Cereb Cortex https://doi.org/10.1093/cercor/bhaa237

5. Zaretskaya N, Fischl B, Reuter M, Renvall V, Polimeni JR (2018) Advantages of cortical surface reconstruction using submillimeter 7 T MEMPRAGE. Neuroimage. 165:11-26 https://doi.org/10.1016/j.neuroimage.2017.09.060

6. Wisse LE, Kuijf HJ, Honingh AM et al (2016) Automated hippocampal subfield segmentation at 7T MRI. AJNR Am J Neuroradiol 37:1050-1057 https://doi.org/10.3174/ajnr.A4659

7. Iglesias JE, Augustinack JC, Nguyen K et al (2015) A computational atlas of the hippocampal formation using ex vivo, ultra-high resolution MRI: Application to adaptive segmentation of in vivo MRI. Neuroimage. 115:117137 https://doi.org/10.1016/j.neuroimage.2015.04.042

8. Solano-Castiella E, Schafer A, Reimer E et al (2011) Parcellation of human amygdala in vivo using ultra high field structural MRI. Neuroimage. 58:741748 https://doi.org/10.1016/j.neuroimage.2011.06.047

9. Saygin ZM, Kliemann D, Iglesias JE et al (2017) High-resolution magnetic resonance imaging reveals nuclei of the human amygdala: manual segmentation to automatic atlas. Neuroimage. 155:370-382 https://doi. org/10.1016/j.neuroimage.2017.04.046

10. von Morze C, Xu D, Purcell DD et al (2007) Intracranial time-of-flight MR angiography at 7T with comparison to 3T. J Magn Reson Imaging 26:900904 https://doi.org/10.1002/jmri.21097

11. Gardener AG, Gowland PA, Francis ST (2009) Implementation of quantitative perfusion imaging using pulsed arterial spin labeling at ultra-high field. Magn Reson Med 61:874-882 https://doi.org/10.1002/mrm.21796

12. Gati JS, Menon RS, Ugurbil K, Rutt BK (1997) Experimental determination of the BOLD field strength dependence in vessels and tissue. Magn Reson Med 38:296-302 https://doi.org/10.1002/mrm.1910380220

13. Triantafyllou C, Hoge RD, Krueger G et al (2005) Comparison of physiological noise at $1.5 \mathrm{~T}, 3 \mathrm{~T}$ and $7 \mathrm{~T}$ and optimization of fMRI acquisition parameters. Neuroimage. 26:243-250 https://doi.org/10.1016/j. neuroimage.2005.01.007

14. Hoff MN, McKinney A, Shellock FG et al (2019) Safety Considerations of 7-T MRI in Clinical Practice. Radiology. 292:509-518 https://doi.org/10.1148/ra diol.2019182742

15. Shellock FG (2020) Reference manual for magnetic resonance safety, implants, and devices. Biomedical Research Publishing Group, California

16. Dula AN, Virostko J, Shellock FG (2014) Assessment of MRI issues at $7 \mathrm{~T}$ for 28 implants and other objects. AJR Am J Roentgenol 202:401-405 https:// doi.org/10.2214/AJR.13.10777

17. Fagan AJ, Bitz AK, Bjorkman-Burtscher IM et al (2021) 7T MR Safety. J Magn Reson Imaging 53:333-346 https://doi.org/10.1002/jmri.27319

18. Caballero-Gaudes C, Reynolds RC (2017) Methods for cleaning the BOLD fMRI signal. Neuroimage. 154:128-149 https://doi.org/10.1016/j.neuroima ge.2016.12.018

19. Hutton C, Josephs $O$, Stadler J et al (2011) The impact of physiological noise correction on fMRI at 7 T. Neuroimage. 57:101-112 https://doi.org/10.1016/j. neuroimage.2011.04.018

20. Nowell M, Miserocchi A, McEvoy AW, Duncan JS (2014) Advances in epilepsy surgery. J Neurol Neurosurg Psychiatry 85:1273-1279 https://doi. org/10.1136/jnnp-2013-307069

21. Opheim G, van der Kolk A, Bloch KM et al (2020) 7T Epilepsy Task Force Consensus Recommendations on the use of $7 \mathrm{~T}$ in Clinical Practice. Neurology. https://doi.org/10.1212/WNL.0000000000011413

22. Obusez EC, Lowe M, Oh SH et al (2018) $7 \mathrm{~T}$ MR of intracranial pathology: preliminary observations and comparisons to $3 \mathrm{~T}$ and 1.5T. Neuroimage. 168 459-476 https://doi.org/10.1016/..neuroimage.2016.11.030

23. Verma G, Delman BN, Balchandani P (2021) UltraHigh Field MR Imaging in Epilepsy. Magn Reson Imaging Clin N Am 29:41-52 https://doi.org/10.1016/j. mric.2020.09.006

24. Feldman RE, Delman BN, Pawha PS et al (2019) 7T MRI in epilepsy patients with previously normal clinical MRI exams compared against healthy controls. PLoS One 14:e0213642 https://doi.org/10.1371/journal.pone.0213642

25. Schlamann M, Maderwald S, Becker W et al (2010) Cerebral cavernous hemangiomas at 7 Tesla: initial experience. Acad Radiol 17:3-6 https://doi. org/10.1016/j.acra.2009.10.001
26. Wang I, Oh S, Blumcke I et al (2020) Value of 7T MRI and post-processing in patients with nonlesional 3T MRI undergoing epilepsy presurgical evaluation. Epilepsia. 61:2509-2520 https://doi.org/10.1111/epi.16682

27. Feldman RE, Rutland JW, Fields MC et al (2018) Quantification of perivascular spaces at 7T: A potential MRI biomarker for epilepsy. Seizure. 54:11-18 https://doi.org/10.1016/j.seizure.2017.11.004

28. Stefanits H, Springer E, Pataraia E et al (2017) Seven-tesla MRI of hippocampal sclerosis: an in vivo feasibility study with histological correlations. Investig Radiol 52:666-671 https://doi.org/10.1097/RLI. 0000000000000388

29. Feldman RE, Marcuse LV, Verma G et al (2020) Seven-tesla susceptibilityweighted analysis of hippocampal venous structures: application to magnetic-resonance-normal focal epilepsy. Epilepsia. 61:287-296 https://doi. org/10.1111/epi.16433

30. Voets NL, Hodgetts CJ, Sen A, Adcock JE, Emir U (2017) Hippocampal MRS and subfield volumetry at 7T detects dysfunction not specific to seizure focus. Sci Rep 7:16138 https://doi.org/10.1038/s41598-017-16046-5

31. Shah P, Bassett DS, Wisse LEM et al (2019) Structural and functional asymmetry of medial temporal subregions in unilateral temporal lobe epilepsy: A 7T MRI study. Hum Brain Mapp 40:2390-2398 https://doi.org/10.1002/hbm.24530

32. Bruschi N, Boffa G, Inglese M (2020) Ultra-high-field 7-T MRI in multiple sclerosis and other demyelinating diseases: from pathology to clinical practice. Eur Radiol Exp 4:59 https://doi.org/10.1186/s41747-020-00186-x

33. Sati P, Oh J, Constable RT et al (2016) The central vein sign and its clinical evaluation for the diagnosis of multiple sclerosis: a consensus statement from the North American Imaging in Multiple Sclerosis Cooperative. Nat Rev Neurol 12:714-722 https://doi.org/10.1038/nrneurol.2016.166

34. Geraldes R, Ciccarelli O, Barkhof F et al (2018) The current role of MRI in differentiating multiple sclerosis from its imaging mimics. Nat Rev Neurol 14:213 https://doi.org/10.1038/nrneurol.2018.39

35. Tallantyre EC, Morgan PS, Dixon JE et al (2009) A comparison of $3 T$ and $7 \mathrm{~T}$ in the detection of small parenchymal veins within MS lesions. Investig Radiol 44:491-494 https://doi.org/10.1097/RLI.0b013e3181b4c144

36. Tallantyre EC, Dixon JE, Donaldson I et al (2011) Ultra-high-field imaging distinguishes MS lesions from asymptomatic white matter lesions. Neurology. 76:534-539 https://doi.org/10.1212/WNL.0b013e31820b7630

37. Hosseini Z, Matusinec J, Rudko DA et al (2018) Morphology-specific discrimination between MS white matter lesions and benign white matter hyperintensities using ultra-high-field MRI. AJNR Am J Neuroradiol 39:14731479 https://doi.org/10.3174/ajnr.A5705

38. Castellaro M, Tamanti A, Pisani Al, Pizzini FB, Crescenzo F, Calabrese M (2020) The Use of the central vein sign in the diagnosis of multiple sclerosis: a systematic review and meta-analysis. Diagnostics (Basel) $10 \mathrm{https}: / /$ doi. org/10.3390/diagnostics10121025

39. Mainero C, Benner T, Radding A et al (2009) In vivo imaging of cortical pathology in multiple sclerosis using ultra-high field MRI. Neurology. 73 : 941-948 https://doi.org/10.1212/WNL.0b013e3181b64bf7

40. Cocozza S, Cosottini M, Signori A et al (2020) A clinically feasible 7-Tesla protocol for the identification of cortical lesions in Multiple Sclerosis. Eur Radiol 30:4586-4594 https://doi.org/10.1007/s00330-020-06803-y

41. Maranzano J, Dadar M, Rudko DA et al (2019) Comparison of multiple sclerosis cortical lesion types detected by multicontrast 3T and 7T MRI. AJNR Am J Neuroradiol 40:1162-1169 https://doi.org/10.3174/ajnr.A6099

42. Treaba CA, Granberg TE, Sormani MP et al (2019) Longitudinal characterization of cortical lesion development and evolution in multiple sclerosis with 7.0-T MRI. Radiology. 291:740-749 https://doi.org/10.1148/radiol.2019181719

43. Louapre C, Treaba CA, Barletta V, Mainero C (2020) Ultra-high field 7 T imaging in multiple sclerosis. Curr Opin Neurol 33:422-429 https://doi.org/1 0.1097 NCO.0000000000000839

44. Inglese M, Fleysher L, Oesingmann N, Petracca M (2018) Clinical applications of ultra-high field magnetic resonance imaging in multiple sclerosis. Expert Rev Neurother 18:221-230 https://doi.org/10.1080/14737175.2018.1433033

45. van der Kolk AG, Hendrikse J, Zwanenburg JJ, Visser F, Luijten PR (2013) Clinical applications of 7 T MRI in the brain. Eur J Radiol 82:708-718 https:// doi.org/10.1016/j.ejrad.2011.07.007

46. Chou IJ, Lim SY, Tanasescu R et al (2018) Seven-tesla magnetization transfer imaging to detect multiple sclerosis white matter lesions. J Neuroimaging 28:183-190 https://doi.org/10.1111/jon.12474

47. Fartaria MJ, Sati P, Todea A et al (2019) Automated detection and segmentation of multiple sclerosis lesions using ultra-high-field MP2RAGE. Investig Radiol 54:356-364 https://doi.org/10.1097/RLI.0000000000000551 
48. Dula AN, Pawate S, Dortch RD et al (2016) Magnetic resonance imaging of the cervical spinal cord in multiple sclerosis at 7T. Mult Scler 22:320-328 https://doi.org/10.1177/1352458515591070

49. Ouellette R, Treaba CA, Granberg T et al (2020) 7 T imaging reveals a gradient in spinal cord lesion distribution in multiple sclerosis. Brain. 143: 2973-2987 https://doi.org/10.1093/brain/awaa249

50. Paek SL, Chung YS, Paek SH et al (2013) Early experience of pre- and postcontrast 7.0T MRI in brain tumors. J Korean Med Sci 28:1362-1372 https:// doi.org/10.3346/jkms.2013.28.9.1362

51. Moenninghoff C, Maderwald S, Theysohn JM et al (2010) Imaging of adult astrocytic brain tumours with 7 T MRI: preliminary results. Eur Radiol 20:704713 https://doi.org/10.1007/s00330-009-1592-2

52. Rutland JW, Delman BN, Gill CM, Zhu C, Shrivastava RK, Balchandani P (2020) Emerging use of ultra-high-field 7T MRI in the study of intracranial vascularity: state of the field and future directions. AJNR Am J Neuroradiol 41:2-9 https://doi.org/10.3174/ajnr.A6344

53. Morrison MA, Lupo JM (2021) 7-T magnetic resonance imaging in the management of brain tumors. Magn Reson Imaging Clin N Am 29:83-102 https://doi.org/10.1016/j.mric.2020.09.007

54. Grabner G, Kiesel B, Wohrer A et al (2017) Local image variance of 7 Tesla $\mathrm{SWl}$ is a new technique for preoperative characterization of diffusely infiltrating gliomas: correlation with tumour grade and IDH1 mutational status. Eur Radiol 27:1556-1567 https://doi.org/10.1007/s00330-016-4451-y

55. Christoforidis GA, Yang M, Abduljalil A et al (2012) "Tumoral pseudoblush" identified within gliomas at high-spatial-resolution ultrahigh-field-strength gradient-echo MR imaging corresponds to microvascularity at stereotactic biopsy. Radiology. 264:210-217 https://doi.org/10.1148/radiol.12110799

56. Radbruch A, Eidel O, Wiestler B et al (2014) Quantification of tumor vessels in glioblastoma patients using time-of-flight angiography at 7 Tesla: a feasibility study. PLoS One 9:e110727 https://doi.org/10.1371/journal.pone. 0110727

57. Grabner G, Nobauer I, Elandt K et al (2012) Longitudinal brain imaging of five malignant glioma patients treated with bevacizumab using susceptibility-weighted magnetic resonance imaging at 7 T. Magn Reson Imaging 30:139-147 https://doi.org/10.1016/j.mri.2011.08.004

58. Regnery S, Knowles BR, Paech D et al (2019) High-resolution FLAIR MRI at 7 Tesla for treatment planning in glioblastoma patients. Radiother Oncol 130: 180-184 https://doi.org/10.1016/j.radonc.2018.08.002

59. Regnery S, Behl NGR, Platt T et al (2020) Ultra-high-field sodium MRI as biomarker for tumor extent, grade and IDH mutation status in glioma patients. Neuroimage Clin 28:102427 https://doi.org/10.1016/j.nicl.2020.102427

60. Hangel G, Cadrien C, Lazen P et al (2020) High-resolution metabolic imaging of high-grade gliomas using 7T-CRT-FID-MRSI. Neuroimage Clin 28: 102433 https://doi.org/10.1016/j.nicl.2020.102433

61. Ladd ME, Bachert P, Meyerspeer M et al (2018) Pros and cons of ultra-highfield MRI/MRS for human application. Prog Nucl Magn Reson Spectrosc 109: 1-50 https://doi.org/10.1016/j.pnmrs.2018.06.001

62. Moser E, Stahlberg F, Ladd ME, Trattnig S (2012) 7-T MR--from research to clinical applications? NMR Biomed 25:695-716 https://doi.org/10.1002/ nbm.1794

63. Li Y, Larson P, Chen AP et al (2015) Short-echo three-dimensional H-1 MR spectroscopic imaging of patients with glioma at 7 Tesla for characterization of differences in metabolite levels. J Magn Reson Imaging 41:1332-1341 https://doi.org/10.1002/jmri.24672

64. An Z, Tiwari V, Ganji SK et al (2018) Echo-planar spectroscopic imaging with dual-readout alternated gradients (DRAG-EPSI) at 7 T: Application for 2hydroxyglutarate imaging in glioma patients. Magn Reson Med 79:18511861 https://doi.org/10.1002/mrm.26884

65. Bogner W, Otazo R, Henning A (2020) Accelerated MR spectroscopic imaging-a review of current and emerging techniques. NMR Biomed:e4314. https://doi.org/10.1002/nbm.4314

66. Hangel G, Jain S, Springer E et al (2019) High-resolution metabolic mapping of gliomas via patch-based super-resolution magnetic resonance spectroscopic imaging at 7T. Neuroimage. 191:587-595 https://doi.org/10.1 016/j.neuroimage.2019.02.023

67. Hingerl L, Strasser B, Moser P et al (2020) Clinical high-resolution 3D-MR spectroscopic imaging of the human brain at 7 T. Investig Radiol 55:239248 https://doi.org/10.1097/RLI.0000000000000626

68. Berrington A, Voets NL, Larkin SJ et al (2018) A comparison of 2hydroxyglutarate detection at 3 and $7 \mathrm{~T}$ with long-TE semi-LASER. NMR Biomed 31 https://doi.org/10.1002/nbm.3886
69. Bisdas S, Chadzynski GL, Braun C et al (2016) MR spectroscopy for in vivo assessment of the oncometabolite 2-hydroxyglutarate and its effects on cellular metabolism in human brain gliomas at 9.4T. J Magn Reson Imaging 44:823-833 https://doi.org/10.1002/jmri.25221

70. Dreher C, Oberhollenzer J, Meissner JE et al (2019) Chemical exchange saturation transfer (CEST) signal intensity at 7T MRI of WHO IV degrees gliomas is dependent on the anatomic location. J Magn Reson Imaging 49: 777-785 https://doi.org/10.1002/jmri.26215

71. Khlebnikov V, van der Kemp WJM, Hoogduin H, Klomp DWJ, Prompers JJ (2019) Analysis of chemical exchange saturation transfer contributions from brain metabolites to the Z-spectra at various field strengths and pH. Sci Rep 9:1089 https://doi.org/10.1038/s41598-018-37295-y

72. Meissner JE, Korzowski A, Regnery S et al (2019) Early response assessment of glioma patients to definitive chemoradiotherapy using chemical exchange saturation transfer imaging at 7 T. J Magn Reson Imaging 50: 1268-1277 https://doi.org/10.1002/jmri.26702

73. Paech D, Dreher C, Regnery S et al (2019) Relaxation-compensated amide proton transfer (APT) MRI signal intensity is associated with survival and progression in high-grade glioma patients. Eur Radiol 29:4957-4967 https:// doi.org/10.1007/s00330-019-06066-2

74. Paech D, Windschuh J, Oberhollenzer J et al (2018) Assessing the predictability of IDH mutation and MGMT methylation status in glioma patients using relaxation-compensated multipool CEST MRI at 7.0 T. NeuroOncology 20:1661-1671 https://doi.org/10.1093/neuonc/noy073

75. Regnery S, Adeberg S, Dreher C et al (2018) Chemical exchange saturation transfer MRI serves as predictor of early progression in glioblastoma patients. Oncotarget 9:28772-28783 https://doi.org/10.18632/oncotarget.2 5594

76. Bulk M, Abdelmoula WM, Nabuurs RJA et al (2018) Postmortem MRI and histology demonstrate differential iron accumulation and cortical myelin organization in early- and late-onset Alzheimer's disease. Neurobiol Aging 62:231-242 https://doi.org/10.1016/j.neurobiolaging.2017.10.017

77. Bulk M, Kenkhuis B, van der Graaf LM, Goeman JJ, Natte R, van der Weerd $L$ (2018) Postmortem T2*-weighted MRI imaging of cortical iron reflects severity of Alzheimer's disease. J Alzheimers Dis 65:1125-1137 https://doi. org/10.3233/JAD-180317

78. Kenkhuis B, Jonkman LE, Bulk M et al (2019) 7T MRI allows detection of disturbed cortical lamination of the medial temporal lobe in patients with Alzheimer's disease. Neuroimage Clin 21:101665 https://doi.org/10.1016/j. nicl.2019.101665

79. van Bergen JM, Li X, Hua J et al (2016) Colocalization of cerebral iron with Amyloid beta in Mild Cognitive Impairment. Sci Rep 6:35514 https://doi. org/10.1038/srep35514

80. van Rooden S, Doan NT, Versluis MJ et al (2015) 7T T(2)*-weighted magnetic resonance imaging reveals cortical phase differences between early- and late-onset Alzheimer's disease. Neurobiol Aging 36:20-26 https:// doi.org/10.1016/j.neurobiolaging.2014.07.006

81. Kerchner GA, Bernstein JD, Fenesy MC et al (2013) Shared vulnerability of two synaptically-connected medial temporal lobe areas to age and cognitive decline: a seven tesla magnetic resonance imaging study. J Neurosci 33:16666-16672 https://doi.org/10.1523/JNEUROSCI.1915-13.2013

82. Kerchner GA, Deutsch GK, Zeineh M, Dougherty RF, Saranathan M, Rutt BK (2012) Hippocampal CA1 apical neuropil atrophy and memory performance in Alzheimer's disease. Neuroimage. 63:194-202 https://doi.org/10.1016/j. neuroimage.2012.06.048

83. Kerchner GA, Hess CP, Hammond-Rosenbluth KE et al (2010) Hippocampal CA1 apical neuropil atrophy in mild Alzheimer disease visualized with 7-T MRI. Neurology. 75:1381-1387 https://doi.org/10.1212/WNL.0b013e3181f73 $6 a 1$

84. Wisse LE, Biessels GJ, Heringa SM et al (2014) Hippocampal subfield volumes at $7 \mathrm{~T}$ in early Alzheimer's disease and normal aging. Neurobiol Aging 35:2039-2045 https://doi.org/10.1016/j.neurobiolaging.2014.02.021

85. Bouvy WH, van Veluw SJ, Kuijf HJ et al (2020) Microbleeds colocalize with enlarged juxtacortical perivascular spaces in amnestic mild cognitive impairment and early Alzheimer's disease: A 7 Tesla MRI study. J Cereb Blood Flow Metab 40:739-746 https://doi.org/10.1177/0271678X19838087

86. Welter ML, Schupbach M, Czernecki V et al (2014) Optimal target localization for subthalamic stimulation in patients with Parkinson disease. Neurology. 82:1352-1361 https://doi.org/10.1212/WNL.0000000000000315

87. Cho ZH, Min HK, Oh SH et al (2010) Direct visualization of deep brain stimulation targets in Parkinson disease with the use of 7-tesla magnetic 
resonance imaging. J Neurosurg 113:639-647 https://doi.org/10.3171/2010.3. JNS091385

88. Abosch A, Yacoub E, Ugurbil K, Harel N (2010) An assessment of current brain targets for deep brain stimulation surgery with susceptibility-weighted imaging at 7 tesla. Neurosurgery. 67:1745-1756 discussion 56. https://doi. org/10.1227/NEU.0b013e3181f74105

89. Patriat R, Cooper SE, Duchin Y et al (2018) Individualized tractography-based parcellation of the globus pallidus pars interna using $7 \mathrm{~T} \mathrm{MRI} \mathrm{in} \mathrm{movement}$ disorder patients prior to DBS surgery. Neuroimage. 178:198-209 https://doi. org/10.1016/j.neuroimage.2018.05.048

90. Plantinga BR, Temel Y, Duchin Y et al (2018) Individualized parcellation of the subthalamic nucleus in patients with Parkinson's disease with 7T MRI. Neuroimage. 168:403-411 https://doi.org/10.1016/j.neuroimage.2016.09.023

91. Plantinga BR, Temel Y, Roebroeck A et al (2014) Ultra-high field magnetic resonance imaging of the basal ganglia and related structures. Front Hum Neurosci 8:876 https://doi.org/10.3389/fnhum.2014.00876

92. Cho ZH, Oh SH, Kim JM et al (2011) Direct visualization of Parkinson's disease by in vivo human brain imaging using $7.0 \mathrm{~T}$ magnetic resonance imaging. Mov Disord 26:713-718 https://doi.org/10.1002/mds.23465

93. Patriat R, Niederer J, Kaplan J et al (2020) Morphological changes in the subthalamic nucleus of people with mild-to-moderate Parkinson's disease: a 7T MRI study. Sci Rep 10:8785 https://doi.org/10.1038/s41598-020-65752-0

94. Poston KL, Ua Cruadhlaoich MAI, Santoso LF et al (2020) Substantia nigra volume dissociates bradykinesia and rigidity from tremor in Parkinson's disease: A 7 Tesla Imaging Study. J Parkinsons Dis 10:591-604 https://doi. org/10.3233/JPD-191890

95. La C, Linortner P, Bernstein JD et al (2019) Hippocampal CA1 subfield predicts episodic memory impairment in Parkinson's disease. Neuroimage Clin 23:101824 https://doi.org/10.1016/..nicl.2019.101824

96. Madai VI, von Samson-Himmelstjerna FC, Bauer M et al (2012) Ultrahighfield MRI in human ischemic stroke--a 7 tesla study. PLoS One 7:e37631 https://doi.org/10.1371/journal.pone.0037631

97. De Cocker LJ, Lindenholz A, Zwanenburg JJ et al (2018) Clinical vascular imaging in the brain at 7T. Neuroimage. 168:452-458 https://doi.org/10.101 6/j.neuroimage.2016.11.044

98. Shao X, Yan L, Ma SJ, Wang K, Wang DJJ (2021) High-Resolution Neurovascular Imaging at 7T: Arterial spin labeling perfusion, 4-dimensional MR angiography, and black blood mr imaging. Magn Reson Imaging Clin N Am 29:53-65 https://doi.org/10.1016/.jmric.2020.09.003

99. Miyazawa $\mathrm{H}$, Natori $\mathrm{T}$, Kameda $\mathrm{H}$ et al (2019) Detecting lenticulostriate artery lesions in patients with acute ischemic stroke using high-resolution MRA at 7 T. Int J Stroke 14:290-297 https://doi.org/10.1177/1747493018806163

100. Kang CK, Park CA, Park CW, Lee YB, Cho ZH, Kim YB (2010) Lenticulostriate arteries in chronic stroke patients visualised by $7 \mathrm{~T}$ magnetic resonance angiography. Int J Stroke 5:374-380 https://doi.org/10.1111/j.1747-4949.201 $0.00464 . x$

101. Yaghi S, Prabhakaran S, Khatri P, Liebeskind DS (2019) Intracranial atherosclerotic disease. Stroke. 50:1286-1293 https://doi.org/10.1161/ STROKEAHA.118.024147

102. Lindenholz A, van der Kolk AG, van der Schaaf IC et al (2020) Intracranial atherosclerosis assessed with 7-T MRl: evaluation of patients with ischemic stroke or transient ischemic attack. Radiology. 295:162-170 https://doi.org/1 0.1148 /radiol.2020190643

103. Lindenholz A, van der Schaaf IC, van der Kolk AG et al (2020) MRI vessel wall imaging after intra-arterial treatment for acute ischemic stroke. AJNR Am J Neuroradiol 41:624-631 https://doi.org/10.3174/ajnr.A6460

104. Zwartbol MHT, Geerlings MI, Ghaznawi R, Hendrikse J, van der Kolk AG, Group U-SS (2019) Intracranial atherosclerotic burden on 7T MRI is associated with markers of extracranial atherosclerosis: the SMART-MR study. AJNR Am J Neuroradiol 40:2016-2022 https://doi.org/10.3174/ajnr.A6308

105. Zhu C, Haraldsson H, Tian B et al (2016) High resolution imaging of the intracranial vessel wall at 3 and 7 T using 3D fast spin echo MRI. MAGMA. 29:559-570 https://doi.org/10.1007/s10334-016-0531-x

106. Majidi S, Sein J, Watanabe M et al (2013) Intracranial-derived atherosclerosis assessment: an in vitro comparison between virtual histology by intravascular ultrasonography, 7T MRI, and histopathologic findings. AJNR Am J Neuroradiol 34:2259-2264 https://doi.org/10.3174/ajnr.A3631

107. Sato T, Matsushige T, Chen B et al (2019) Wall contrast enhancement of thrombosed intracranial aneurysms at 7T MRI. AJNR Am J Neuroradiol 40: 1106-1111 https://doi.org/10.3174/ajnr.A6084
108. Wrede KH, Dammann P, Monninghoff C et al (2014) Non-enhanced MR imaging of cerebral aneurysms: 7 Tesla versus 1.5 Tesla. PLoS One 9:e84562 https://doi.org/10.1371/journal.pone.0084562

109. Wrede KH, Matsushige T, Goericke SL et al (2017) Non-enhanced magnetic resonance imaging of unruptured intracranial aneurysms at 7 Tesla: comparison with digital subtraction angiography. Eur Radiol 27:354-364 https://doi.org/10.1007/s00330-016-4323-5

110. Van Essen DC, Ugurbil K, Auerbach E et al (2012) The Human Connectome Project: a data acquisition perspective. Neuroimage. 62:2222-2231 https:// doi.org/10.1016/.neuroimage.2012.02.018

111. Setsompop K, Kimmlingen R, Eberlein E et al (2013) Pushing the limits of in vivo diffusion MRI for the Human Connectome Project. Neuroimage. 80: 220-233 https://doi.org/10.1016/j.neuroimage.2013.05.078

112. Foo TKF, Tan ET, Vermilyea ME et al (2020) Highly efficient head-only magnetic field insert gradient coil for achieving simultaneous high gradient amplitude and slew rate at 3.0T (MAGNUS) for brain microstructure imaging. Magn Reson Med 83:2356-2369 https://doi.org/10.1002/mrm.2 8087

113. Winkler SA, Schmitt F, Landes $H$ et al (2018) Gradient and shim technologies for ultra high field MRI. Neuroimage. 168:59-70 https://doi. org/10.1016/..neuroimage.2016.11.033

114. Stockmann JP, Wald LL (2018) In vivo BO field shimming methods for MRI at 7T. Neuroimage. 168:71-87 https://doi.org/10.1016/.neuroimage.2017.06.013

115. Polimeni JR, Wald LL (2018) Magnetic resonance imaging technologybridging the gap between noninvasive human imaging and optical microscopy. Curr Opin Neurobiol 50:250-260 https://doi.org/10.1016/j. conb.2018.04.026

116. Davids M, Guerin B, Vom Endt A, Schad LR, Wald LL (2019) Prediction of peripheral nerve stimulation thresholds of MRI gradient coils using coupled electromagnetic and neurodynamic simulations. Magn Reson Med 81:686$701 \mathrm{https} / / /$ doi.org/10.1002/mrm.27382

117. Davids M, Guerin B, Klein V, Wald LL (2020) Optimization of MRI gradient coils with explicit peripheral nerve stimulation constraints. IEEE Trans Med Imaging https://doi.org/10.1109/TMI.2020.3023329

118. Tan ET, Hua Y, Fiveland EW et al (2020) Peripheral nerve stimulation limits of a high amplitude and slew rate magnetic field gradient coil for neuroimaging. Magn Reson Med 83:352-366 https://doi.org/10.1002/mrm.2 7909

119. Meyer CH, Hu BS, Nishimura DG, Macovski A (1992) Fast spiral coronary artery imaging. Magn Reson Med 28:202-213 https://doi.org/10.1002/mrm.1 910280204

120. Frahm J, Voit D, Uecker M (2019) Real-time magnetic resonance imaging: radial gradient-echo sequences with nonlinear inverse reconstruction. Investig Radiol 54:757-766 https://doi.org/10.1097/RLI.0000000000000584

121. Bilgic B, Gagoski BA, Cauley SF et al (2015) Wave-CAIPI for highly accelerated 3D imaging. Magn Reson Med 73:2152-2162 https://doi.org/1 $0.1002 / \mathrm{mrm} .25347$

122. Cauley SF, Setsompop K, Bilgic B, Bhat H, Gagoski B, Wald LL (2017) Autocalibrated wave-CAIPI reconstruction; Joint optimization of k-space trajectory and parallel imaging reconstruction. Magn Reson Med 78:10931099 https://doi.org/10.1002/mrm.26499

123. Polak D, Cauley S, Huang SY et al (2019) Highly-accelerated volumetric brain examination using optimized wave-CAIPI encoding. J Magn Reson Imaging 50:961-974 https://doi.org/10.1002/jmri.26678

124. Conklin J, Longo MGF, Cauley SF et al (2019) Validation of highly accelerated wave-CAIPI SWI compared with conventional SWI and T2*weighted gradient recalled-echo for routine clinical brain MRI at 3T. AJNR Am J Neuroradiol 40:2073-2080 https://doi.org/10.3174/ajnr.A6295

125. Goncalves Filho ALM, Conklin J, Longo MGF et al (2020) Accelerated postcontrast wave-CAIPI T1 SPACE achieves equivalent diagnostic performance compared with standard T1 SPACE for the detection of brain metastases in clinical 3T MRI. Front Neurol 11:587327 https://doi.org/10.3389/fneur.2020. 587327

126. Longo MGF, Conklin J, Cauley SF et al (2020) Evaluation of ultrafast waveCAIPI MPRAGE for visual grading and automated measurement of brain tissue volume. AJNR Am J Neuroradiol 41:1388-1396 https://doi.org/10.31 74/ajnr.A6703

127. McNab JA, Edlow BL, Witzel T et al (2013) The human connectome project and beyond: initial applications of $300 \mathrm{mT} / \mathrm{m}$ gradients. Neuroimage. 80: 234-245 https://doi.org/10.1016/..neuroimage.2013.05.074 
128. Fan Q, Nummenmaa A, Witzel T et al (2014) Investigating the capability to resolve complex white matter structures with high b-value diffusion magnetic resonance imaging on the MGH-USC Connectom scanner. Brain Connect 4:718-726 https://doi.org/10.1089/brain.2014.0305

129. Fan Q, Nummenmaa A, Polimeni JR et al (2017) Hlgh b-value and high Resolution Integrated Diffusion (HIBRID) imaging. Neuroimage. 150:162-176 https://doi.org/10.1016/j.neuroimage.2017.02.002

130. Zhang H, Schneider T, Wheeler-Kingshott CA, Alexander DC (2012) NODDI: practical in vivo neurite orientation dispersion and density imaging of the human brain. Neuroimage. 61:1000-1016 https://doi.org/10.1016/j.neuroima ge.2012.03.072

131. Assaf Y, Blumenfeld-Katzir T, Yovel Y, Basser PJ (2008) AxCaliber: a method for measuring axon diameter distribution from diffusion MRI. Magn Reson Med 59:1347-1354 https://doi.org/10.1002/mrm.21577

132. Alexander DC, Hubbard PL, Hall MG et al (2010) Orientationally invariant indices of axon diameter and density from diffusion MRI. Neuroimage. 52: 1374-1389 https://doi.org/10.1016/j.neuroimage.2010.05.043

133. Dyrby TB, Sogaard LV, Hall MG, Ptito M, Alexander DC (2013) Contrast and stability of the axon diameter index from microstructure imaging with diffusion MRI. Magn Reson Med 70:711-721 https://doi.org/10.1002/mrm.24 501

134. Huang SY, Nummenmaa A, Witzel T et al (2015) The impact of gradient strength on in vivo diffusion MRI estimates of axon diameter. Neuroimage. 106:464-472 https://doi.org/10.1016/j.neuroimage.2014.12.008

135. Fan Q, Nummenmaa A, Wichtmann B et al (2018) Validation of diffusion MRI estimates of compartment size and volume fraction in a biomimetic brain phantom using a human MRI scanner with $300 \mathrm{mT} / \mathrm{m}$ maximum gradient strength. Neuroimage. 182:469-478 https://doi.org/10.1016/j. neuroimage.2018.01.004

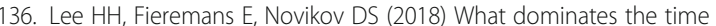
dependence of diffusion transverse to axons: Intra- or extra-axonal water? Neuroimage. 182:500-510 https://doi.org/10.1016/j.neuroimage.2017.12.038

137. Veraart J, Nunes D, Rudrapatna U et al (2020) Nonivasive quantification of axon radii using diffusion MRI. Elife. 9 https://doi.org/10.7554/eLife.49855

138. Huang SY, Tian Q, Fan Q et al (2020) High-gradient diffusion MRI reveals distinct estimates of axon diameter index within different white matter tracts in the in vivo human brain. Brain Struct Funct 225:1277-1291 https:// doi.org/10.1007/s00429-019-01961-2

139. Fan Q, Nummenmaa A, Witzel T et al (2020) Axon diameter index estimation independent of fiber orientation distribution using high-gradient diffusion MRI. Neuroimage 222:117197 https://doi.org/10.1016/j.neuroima ge. 2020.117197

140. Huang SY, Tobyne SM, Nummenmaa A et al (2016) Characterization of axonal disease in patients with multiple sclerosis using high-gradientdiffusion MR imaging. Radiology:151582 https://doi.org/10.1148/radiol.20161 51582

141. Yu F, Fan Q, Tian Q et al (2019) Imaging G-ratio in multiple sclerosis using high-gradient diffusion MRI and macromolecular tissue volume. AJNR Am J Neuroradiol 40:1871-1877 https://doi.org/10.3174/ajnr.A6283

142. Ngamsombat C, Tian Q, Fan Q et al (2020) Axonal damage in the optic radiation assessed by white matter tract integrity metrics is associated with retinal thinning in multiple sclerosis. Neuroimage Clin 27:102293 https://doi. org/10.1016/j.nicl.2020.102293

143. Evangelou N, Esiri MM, Smith S, Palace J, Matthews PM (2000) Quantitative pathological evidence for axonal loss in normal appearing white matter in multiple sclerosis. Ann Neurol 47:391-395

144. Huang SY, Fan Q, Machado N et al (2019) Corpus callosum axon diameter relates to cognitive impairment in multiple sclerosis. Ann Clin Transl Neurol 6:882-892 https://doi.org/10.1002/acn3.760

145. Fan Q, Tian Q, Ohringer NA et al (2019) Age-related alterations in axonal microstructure in the corpus callosum measured by high-gradient diffusion MRI. Neuroimage. 191:325-336 https://doi.org/10.1016/j.neuroimage.2019.02. 036

146. Salat DH, Tuch DS, Greve DN et al (2005) Age-related alterations in white matter microstructure measured by diffusion tensor imaging. Neurobiol Aging 26:1215-1227 https://doi.org/10.1016/j.neurobiolaging.2004.09.017

147. Tan ET, Shih RY, Mitra J et al (2020) Oscillating diffusion-encoding with a high gradient-amplitude and high slew-rate head-only gradient for human brain imaging. Magn Reson Med 84:950-965 https://doi.org/10.1002/mrm.2 8180
148. Yang G, Tian Q, Leuze C, Wintermark M, McNab JA (2018) Double diffusion encoding MRI for the clinic. Magn Reson Med 80:507-520 https://doi.org/1 0.1002/mrm.27043

149. Westin CF, Knutsson H, Pasternak O et al (2016) Q-space trajectory imaging for multidimensional diffusion MRI of the human brain. Neuroimage. 135: 345-362 https://doi.org/10.1016/j.neuroimage.2016.02.039

150. Tax CM, Szczepankiewicz F, Nilsson M, Jones DK (2019) The dotcompartment revealed? Diffusion MRI with ultra-strong gradients and spherical tensor encoding in the living human brain. bioRxiv https://doi. org/10.1101/584730

151. Szczepankiewicz F, Lasic S, van Westen D et al (2015) Quantification of microscopic diffusion anisotropy disentangles effects of orientation dispersion from microstructure: applications in healthy volunteers and in brain tumors. Neuroimage. 104:241-252 https://doi.org/10.1016/j.neuroima ge.2014.09.057

152. Lewis LD, Setsompop K, Rosen BR, Polimeni JR (2016) Fast fMRI can detect oscillatory neural activity in humans. Proc Natl Acad Sci U S A 113:E6679E6E85 https://doi.org/10.1073/pnas.1608117113

153. Lewis LD, Setsompop K, Rosen BR, Polimeni JR (2018) Stimulus-dependent hemodynamic response timing across the human subcortical-cortical visual pathway identified through high spatiotemporal resolution 7T fMRI. Neuroimage. 181:279-291 https://doi.org/10.1016/j.neuroimage.2018.06.056

\section{Publisher's Note}

Springer Nature remains neutral with regard to jurisdictional claims in published maps and institutional affiliations.

\section{Submit your manuscript to a SpringerOpen ${ }^{\circ}$ journal and benefit from:}

- Convenient online submission

- Rigorous peer review

- Open access: articles freely available online

High visibility within the field

- Retaining the copyright to your article

Submit your next manuscript at $>$ springeropen.com 